\title{
Deformation and Interaction of Droplet Pairs in a Microchannel Under ac Electric Fields
}

\author{
Xiaodong Chen, ${ }^{1}$ Yongxin Song, ${ }^{2}$ Dongqing $\mathrm{Li}^{2,3}$ and Guoqing $\mathrm{Hu}^{1, *}$ \\ ${ }^{1}$ State Key Laboratory of Nonlinear Mechanics, Institute of Mechanics, \\ Chinese Academy of Sciences, Beijing 100190, China \\ ${ }^{2}$ Department of Marine Engineering, Dalian Maritime University, Dalian 116026, China \\ ${ }^{3}$ Department of Mechanical and Mechatronics Engineering, University of Waterloo, \\ Waterloo, Ontario N2L 3G1, Canada
}

(Received 5 December 2014; revised manuscript received 10 May 2015; published 7 August 2015)

\begin{abstract}
The deformation and interaction of a droplet pair in an electric field determine the success of droplet coalescence. Electric intensity and initial droplet separation are crucial parameters in this process. In this work, a combined theoretical and numerical analysis is performed to study the electrohydrodynamics of confined droplet pairs in a rectangular microchannel under ac electric fields. We develop a theoretical model to predict the relationship between critical electric intensity and droplet separation. A geometrical model relating the initial droplet separation to the cone angle is also established to determine the critical separation for partial coalescence. These models are validated by comparisons with existing experimental observations. According to the initial separation and electric intensity, five regimes of droplet interactions are classified by direct numerical simulations, namely noncoalescence, coalescence, partial coalescence, ejection after coalescence, and ejection with partial coalescence. According to their controlling mechanisms, the five regimes are distinguished by three well-defined boundaries. The detailed dynamics of the partial coalescence phenomenon is resolved when the droplet separation exceeds the critical value. A dynamic liquid bridge between the droplets is sustained by the competition between surface tension and electric stress. The dynamics of ejected microjets at the exterior ends are also addressed to show their responses to the oscillating electric field. The full understanding of the droplet dynamics under electric fields can be used to predict the droplet fusion behaviors and thus to facilitate the design of droplet-based microfluidic devices.
\end{abstract}

DOI: 10.1103/PhysRevApplied.4.024005

\section{INTRODUCTION}

Droplet fusion is one of the fundamental functions in droplet-based microfluidic devices, for example, to precisely control the mixing of regents in space and time for microreaction applications [1]. Passive fusion relies on channel properties to bring droplets into close contact [2,3], while active fusion relies on external forces to coalesce the droplets [1]. Electric fields are widely used as active techniques to trigger the coalescence in microchannels [4-6]. Droplets can be polarized in electric fields, resulting in dielectrophoretic forces at the oil-water interfaces. Two close droplet interfaces can then attract each other to bring the adjacent points to contact and eventually merge themselves into one. This process induced by electrostatic attraction forces is called electrocoalescence $[7,8]$. There are many applied studies $[4,9,10]$ on the electrocoalescence of two neighboring droplets separated by a thin film in microchannels. This paper explores situations with considerable separations between the droplets.

Taylor [11] theoretically and experimentally studied the deformation and disintegration of a spherical water droplet

*Corresponding author. guoqing.hu@imech.ac.cn situated in a direct-current (dc) electric field. The droplet becomes unstable when the electric intensity reaches a well-defined critical value. The ends of the droplets develop obtuse-angled conical points from which axial jets are emitted. The liquid cone is commonly known as a Taylor cone [12]. The disruption point was predicted to be reached when $E\left(R_{0} / \sigma\right)^{1 / 2}=1.62$, where $E$ is the electric intensity, $R_{0}$ is the undistorted droplet radius, and $\sigma$ is the surface tension (all in electrostatic units). This critical value is in good agreement with the experimental observations of Nolan [13] and Macky [14]. When two droplets are close in an electric field, the interaction of the opposite polarization charges enhances the electric field between the adjacent surfaces of the droplets. Latham and Roxburgh [15] extended Taylor's analysis to droplet pairs by introducing a field-enhancement parameter calculated by Davis [16]. The predicted critical electric intensities for different droplet separations, the measured curvatures, and elongations of droplets were in good agreement with experiments.

Chabert et al. [5] experimentally studied the electrocoalescence of microfluidic droplets with diameters comparable to that of a round channel. They found that $\mathrm{dc}$ electric fields could not induce coalescence, but alternatingcurrent (ac) electric fields could. This is because, under a dc field, all ions and charge carriers quickly move to the 
insulating material between the electrodes and the emulsion. The voltage drop is mainly distributed across the insulating material, and no electric field is in the emulsion. In contrast, under an ac field, the ions and charge carriers do not have time to move to the insulating material in the duration of one half period of the voltage. The electric field is then mainly distributed in the emulsion [17]. By applying an ac electric field, Chabert et al. [5] obtained the regime diagram of electrocoalescence as a function of the amplitude and frequency of the voltage. The lower boundary between the noncoalescence and coalescence regimes was found to be independent of the frequency. An upper boundary showed a regime where the droplets contact to form an unstable liquid bridge between them. The liquid bridge broke to cause the droplets to repel one another. Recently, Thiam et al. [18] investigated the regime diagram of water droplet pairs in hexadecane as a function of droplet separation and ac field intensity. Three regimes were discovered, namely stable (noncoalescence), coalescence, and partial coalescence. The latter regime was similar to the formation of the liquid bridge observed by Chabert et al. [5]. Other than droplet recoil, Thiam et al. [18] observed that the liquid bridge could be almost stable for droplets with ultralow conductivity.

The recoil phenomenon of droplet interaction also occurred in different configurations [19,20]. Bird et al. [21] showed that the transition between coalescence and recoil is determined by the relationship of two principle curvatures of the liquid neck region where the cones of two droplets contact. If the negative curvature across the neck is much larger than the positive curvature around the neck, the neck expands radially under the capillary force to merge the droplets. Reversely, the neck shrinks and breaks eventually to cause droplet recoil [21]. Geometrically, the coalescence behavior of two droplets depends on the angle of the cones when the droplets just contact. Bird et al. [21] developed a surface energy model to predict the critical angle. The result agreed well with experimental measurements.

In an early numerical study, Brazier-Smith et al. [22] considered conductive droplets in a nonconducting medium under a strong electrical field. They showed two types of interaction of the droplets, depending on the ratios of droplet separation and radius. For a ratio less than about 1.2 , the droplets deform and their near interfaces make contact. For a ratio greater than about 1.2, the droplets deform into conical profiles with concavities at the adjacent poles. Subsequent issuance of a liquid jet from the adjacent poles was not able to be resolved in their simulations. Sozou [23] used the leaky dielectric model [24] to analyze the flow field generated by a uniform electrostatic field in and about a pair of nondeformable liquid droplets. The flow field over the droplet surfaces due to the tangential electric stresses was expressed in terms of bispherical harmonics. Baygents et al. [25] simulated the motion of two axisymmetric droplets in a uniform electric field using an integral equation method and the leaky dielectric model. Different from perfect dielectrics, in which the electrical stress acts only normal to the interfaces, the leaky dielectric model shows that the conduction process leads to tangential electrical stresses on the droplet interface. The tangential stresses cause steady circulation in and about the droplet. Three classes of behaviors were observed, depending on the ratios of dielectric constants and electrical conductivities of the droplet and the ambient fluid [25] — the droplets first deform oblately and then translate together; the droplets deform prolately and translate together; or the droplets deform prolately and move apart. More recently, Mohammadi et al. [26] numerically simulated the approaching of two droplets under an electric field using a volume-of-fluid approach. The electric dipole-dipole force between droplets was considered as a body force acting on droplets through a mathematical model [27], but no electric-induced interfacial deformation was resolved.

Much useful information has been obtained about the electrohydrodynamics of a droplet pair, however, the combined effects of droplet separation and electric intensity under an oscillating electric field need to be explored further. Our work focuses on the effects of these key parameters on the deformation and interaction of a droplet pair, especially the boundaries among different regimes. A theoretical model is established to predict the critical electric intensity for droplet coalescence. Good agreements are obtained with the existing experimental study. Threedimensional direct numerical simulations are performed to study the detailed evolution of droplet interaction. Five regimes, divided by three well-defined boundaries, are identified in terms of initial droplet separation and electric intensity. The dynamics of the liquid bridge between droplets and tip streaming at the droplet ends are also addressed to reveal the underlying mechanics.

\section{THEORETICAL ANALYSIS}

\section{A. Problem statement}

Following the experiments of Thiam et al. [18], we examine two equal-sized and uncharged droplets under an ac electric field. Figure 1(a) shows that the droplets move with the surrounding fluid along the centerline of a microchannel. The dispersed phase is Milli-Q water and the continuous phase is hexadecane [18], which is a commonly used combination in droplet-based microfluidic devices. The droplets are confined by the top and bottom walls to adapt cheese-wheel-like shapes. Due to the lubrication effect, thin films exist between the droplet interfaces and the walls to avoid contacts. The undisturbed ac electric field is uniformly distributed along the flow direction with a frequency of $10 \mathrm{kHz}$. The electric potential across the considered domain is $V_{t}$. The electric intensity $E_{t}$ along the length of the microchannel is thus $-V_{t} / L$. The root-mean-square value of $V_{t}, V=\sqrt{2} V_{\max } / 2$, is used for evaluating the electric field, where $V_{\max }$ is the maximum 


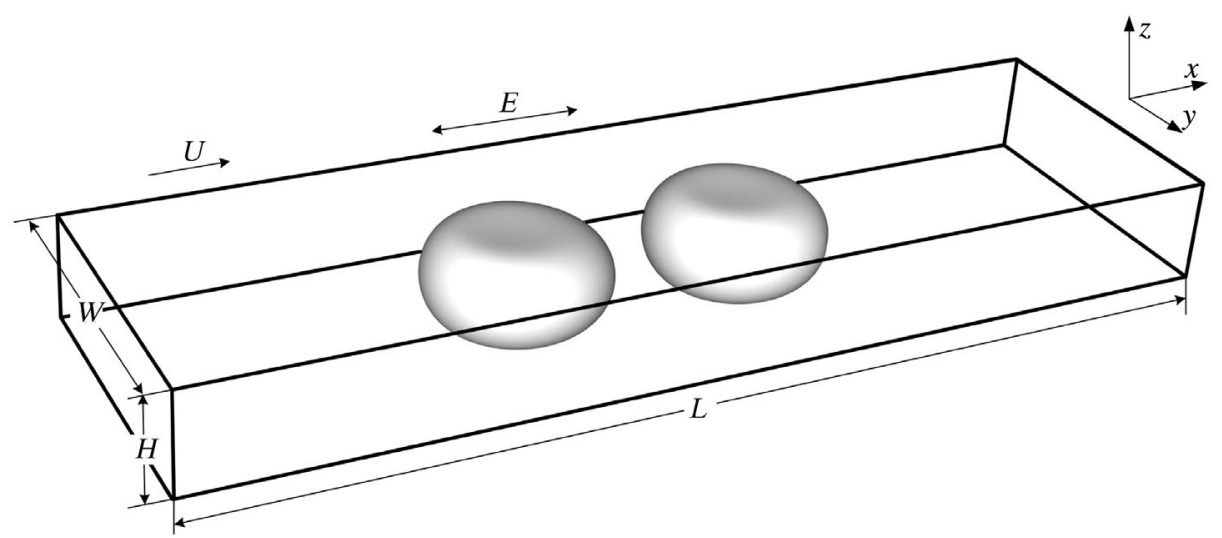

(a)

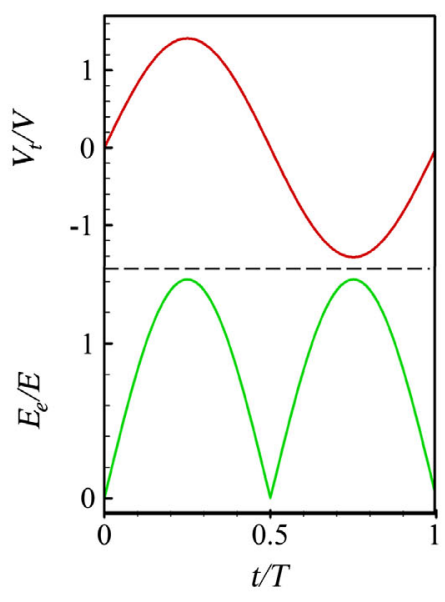

(b)

FIG. 1 (a) Schematic diagram of a droplet pair in a pressure-driven flow through a microchannel with rectangular cross section. (b) Evolution of the electric potential and effective electric intensity in one ac period.

electric potential. Because the charges aggregated near the two droplet surfaces always attract each other, the effective electric intensity, $E_{e}=\left|E_{t}\right|$, should be used to describe the interaction of the droplets. Figure 1(b) shows the evolutions of $V_{t}$ and $E_{e}$ in a period of $T$, where $E=V / L$. The variation of $E_{e}$ shows a pulsating pattern with a frequency of twice the ac field.

Dimensional analysis gives eight dimensionless numbers: Ohnesorge number, electric Bond number, dimensionless electrical conductivity, dimensionless mean velocity, and the ratios of the densities, viscosities, relative permittivities, and electric conductivities of water and oil. They are defined as $\mathrm{Oh}=\mu_{w} /\left(\rho_{w} R_{0} \sigma\right)^{1 / 2}, B_{e}=\varepsilon_{0} V^{2} /$ $\left(R_{0} \sigma\right), K=\left[\rho_{w} R_{0}^{3} k_{w}^{2} /\left(\sigma \varepsilon_{0}^{2}\right)\right]^{1 / 2}, U_{m}=Q / \pi\left[\rho_{w} /\left(R_{0}^{3} \sigma\right)\right]^{1 / 2}$, $\alpha=\rho_{w} / \rho_{o}, \lambda=\mu_{w} / \mu_{o}, \gamma=\varepsilon_{w} / \varepsilon_{o}$, and $\beta=k_{w} / k_{o}$, respectively, where the density, viscosity, electric permittivity, and conductivity of water are denoted by $\rho_{w}, \mu_{w}, \varepsilon_{w}$, and $k_{w}$, respectively, and those of oil have a subscript of $o . R_{0}$ is the droplet diameter measured from the top view, $\sigma$ is the surface tension, $Q$ is the flow rate, and $\varepsilon_{0}$ is the permittivity in a vacuum. Table I lists the physical parameters of a $0.01 M \mathrm{NaCl}$ solution and hexadecane. $H, W$, and $L$ denote the height, width, and length of the microchannel, respectively. As in most microfluidic devices, the Reynolds number based on the channel height is small in this paper, about 0.15 . The presence of flow allows oil films to form between the droplets and the walls. This provides a cushion effect which allows droplets to avoid contact with walls, but has negligible effect on the deformation and interaction of the droplet pair.

\section{B. Prediction of critical electric intensity}

This problem is similar to that in Ref. [15] for a pair of spherical droplets. However, droplets confined by the walls of the microchannel have to adapt cheese-wheel-like shapes. We introduce a modified geometrical assumption to extend the theoretical frame of Ref. [15]. Figure 2(a) illustrates that two identical uncharged droplets are flowing in a microchannel with the ambient fluid. Since the Reynolds number of the flow is much less than 1 , the effect of the flow on the droplet deformation is weak. It is then reasonable to neglect the effect of flow in the theoretical prediction. Droplet shapes in the $x$-y cross section are circles with undistorted radii $R_{0}$. The initial separation of the adjacent points $A$ and $C$ is $X_{0}$. $D$ is the rear point. The droplets are polarized when a uniform electric field is applied parallel to the line of droplet centers [Fig. 2(b)]. The attractive interactions of the polarization charges bring $A$ and $C$ close and thus cause extra droplet deformations. The droplets are assumed to retain an elliptical cross section in the $x-y$ plane until disintegration. The droplet shape is then given by $x^{2} a^{-2}+y^{2} b^{-2}=1$ with respect to each droplet centroid, where $a$ and $b$ are the lengths of the semimajor and semiminor axes, respectively. The product of $a$ and $b$ is $R_{0}^{2}$ due to the conservation of surface area of the elliptical shape. Using the equations of equilibrium at $A$ (or $C$ ) and $B$, Latham and Roxburgh [15] obtained the link between $E$ and $a / b$ for spheroidal droplets, which deform into axisymmetric spheroids in electric fields. The deformation of a flattened droplet is three-dimensional and complicated to predict. To overcome

TABLE I. Physical parameters used in the present study.

\begin{tabular}{|c|c|c|c|c|c|c|c|c|c|c|c|c|c|}
\hline $\begin{array}{l}\rho_{w} \\
\left(\mathrm{~kg} / \mathrm{m}^{3}\right)\end{array}$ & $\begin{array}{c}\rho_{o} \\
\left(\mathrm{~kg} / \mathrm{m}^{3}\right)\end{array}$ & $\begin{array}{c}\mu_{w} \\
(\mathrm{mPa} s)\end{array}$ & $\begin{array}{c}\mu_{o} \\
(\mathrm{mPas})\end{array}$ & $\varepsilon_{w}$ & $\varepsilon_{o}$ & $\begin{array}{c}k_{w} \\
(\mathrm{~S} / \mathrm{m})\end{array}$ & $\begin{array}{c}k_{o} \\
(\mathrm{~S} / \mathrm{m})\end{array}$ & $\begin{array}{c}R_{0} \\
(\mu \mathrm{m})\end{array}$ & $\begin{array}{c}\sigma \\
(\mathrm{mN} / \mathrm{m})\end{array}$ & $\begin{array}{c}Q \\
(\mathrm{~mL} / \mathrm{h})\end{array}$ & $\begin{array}{c}H \\
(\mu \mathrm{m})\end{array}$ & $\begin{array}{c}W \\
(\mu \mathrm{m})\end{array}$ & $\begin{array}{c}L \\
(\mathrm{~mm})\end{array}$ \\
\hline 998 & 768 & 1 & 3 & 80 & 2 & $1.2 \times 10^{-3}$ & $1.5 \times 10^{-15}$ & 20 & 4 & 0.11 & 23 & 69 & 230 \\
\hline
\end{tabular}



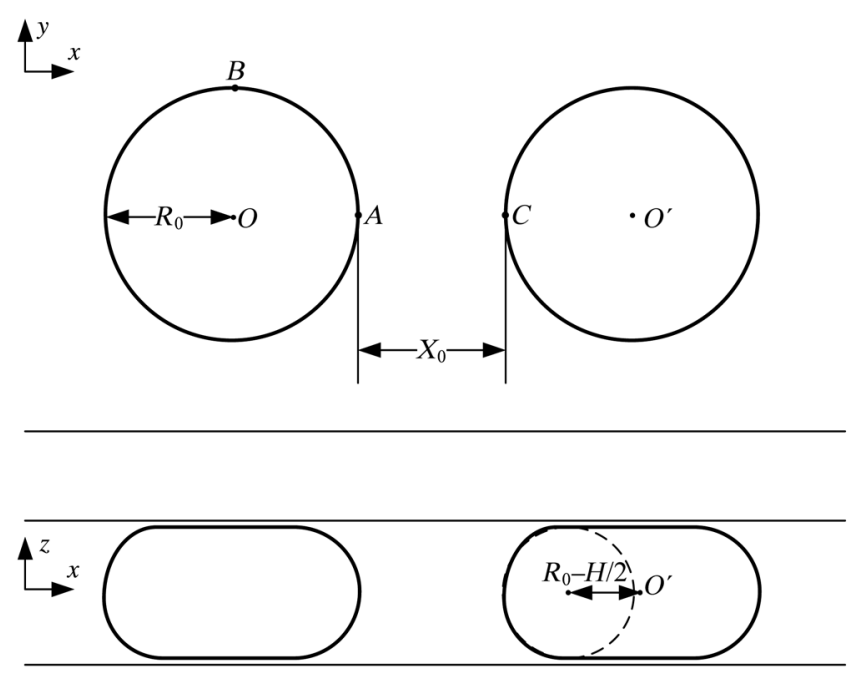

(a)
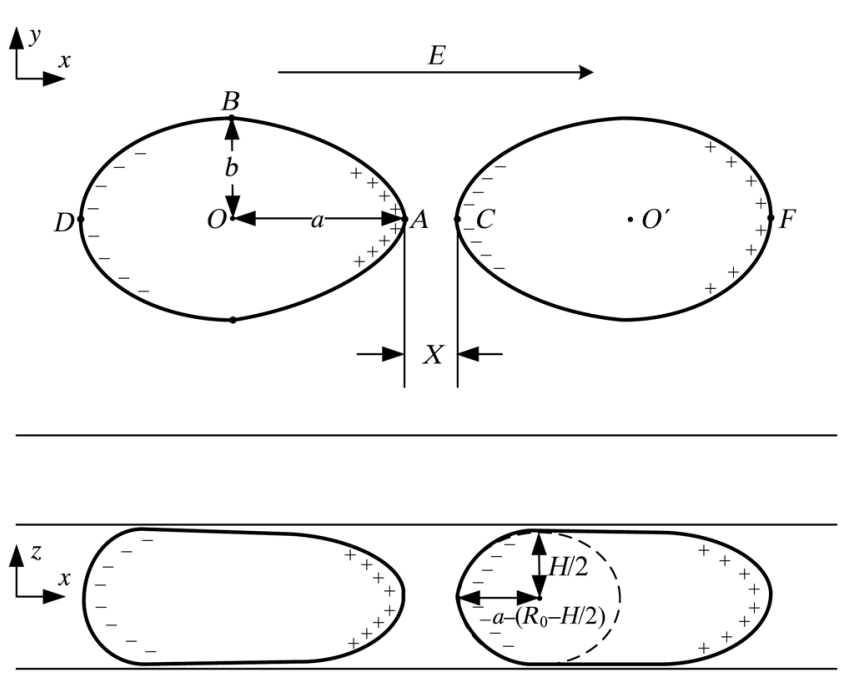

(b)

FIG. 2. Schematics of a droplet pair situated in a microchannel. (a) Initial shapes of droplets in $x-y$ and $y-z$ cross sections. (b) Deformation of droplets under an electric field. Note that the schematics are generated based on our numerical simulation in Sec. III.

this difficulty, we introduce an additional assumption that the profiles of the adjacent interfaces are elliptical in the $x-z$ cross section through the droplet centroids. Owing to the confinement, the length of the semiminor axis is fixed to be $H / 2$. The length of the semimajor axis is coupled with the deformation in the $x-y$ cross section to be $a-\left(R_{0}-H / 2\right)$ [see Fig. 2(b)]. The shape of a deformed droplet in the $x-z$ cross section through point $A$ (or $C$ ) is then given by

$$
\frac{x^{2}}{\left[a-\left(R_{0}-H / 2\right)\right]^{2}}+\frac{z^{2}}{(H / 2)^{2}}=1
$$

Similarly, the shape of a deformed droplet in the $y-z$ cross section through point $B$ is given by

$$
\frac{y^{2}}{\left[b-\left(R_{0}-H / 2\right)\right]^{2}}+\frac{z^{2}}{(H / 2)^{2}}=1
$$

The normal stress at the droplet interface due to surface tension is $\sigma\left(r_{1}^{-1}+r_{2}^{-1}\right)$, where $\sigma$ is surface tension, $r_{1}$ is the principal radius of the curvature of the ellipse, and $r_{2}$ is the other radius of curvature. From the shape described by Eqs. (1) and (2), the expressions of $\left(r_{1}^{-1}+r_{2}^{-1}\right)$ can be obtained analytically. At point $A$,

$$
r_{1}^{-1}+r_{2}^{-1}=a b^{-2}+\left[a-\left(R_{0}-H / 2\right)\right](H / 2)^{-2},
$$

and at point $B$,

$$
r_{1}^{-1}+r_{2}^{-1}=b a^{-2}+\left[b-\left(R_{0}-H / 2\right)\right](H / 2)^{-2},
$$

The electric intensity at the point $A$ and $C$ is assumed to be equal to that of single droplets [11] multiplied by a factor $\Sigma$, which is the enhancement of the electric field because of the mutual interaction of the polarization charges [15]. The normal stress at the point $A$ or $C$ due to the electric field is then [15]

$$
\tau_{a}=\frac{\Sigma^{2} E^{2} \varepsilon_{o} \varepsilon_{0} a^{2}}{18 b^{2}\left(1-e^{2}\right) I^{2}},
$$

where the eccentricity $e=\left(1-b^{2} / a^{2}\right)^{1 / 2}$, and $I=1 / 2 e^{-3} \ln [(1+e) /(1-e)]-e^{-2}$. The value of $\Sigma$ was calculated by Davis [16] as a function of $X / r$, where $r$ is the radius of two identical rigid spheres. The approximation of $r=R_{0}$ was proved to be reasonable by Latham and Roxburgh [15] and is used in the subsequent calculation. Under the assumption that distance between the droplet centroids does not change during the interaction, the separation $X$ of the adjacent points $A$ and $C$ can be then expressed as [15]

$$
X=X_{0}-2\left(a-R_{0}\right) .
$$

The relationship between $a / b$ and $\Sigma$ can be derived as

$$
\Sigma=f\left(X / R_{0}\right)=f\left[X_{0} / R_{0}-2(a / b)^{1 / 2}+2\right],
$$

where the $a b=R_{0}^{2}$ is used according to the elliptical assumption. Note that for spheroidal droplets, the power of $(a / b)$ in Eq. (7) is $2 / 3$ due to the volume conservation. On the other hand, there is no normal stress at the point $B$ since the electric field is perpendicular to the direction of normal stress.

Following Ref. [11], the equation of equilibrium at $A$ (or $C$ ) is given by

$$
\sigma\left\{a b^{-2}+\left[a-\left(R_{0}-H / 2\right)\right](H / 2)^{-2}\right\}-p=\tau_{a},
$$

where $p$ is the difference between the internal and external pressure. The equation of equilibrium at $B$ is given by 


$$
\sigma\left\{b a^{-2}+\left[b-\left(R_{0}-H / 2\right)\right](H / 2)^{-2}\right\}-p=0 .
$$

Combining Eqs. (8) and (9) gives

$$
\sigma\left[a b^{-2}-b a^{-2}+(a-b)(H / 2)^{-2}\right]=\frac{\Sigma^{2} E^{2} \varepsilon_{0} \varepsilon_{o} a^{2}}{18 b^{2}\left(1-e^{2}\right) I^{2}},
$$

which can be rewritten to be

$$
\begin{aligned}
E / E_{0}= & 18^{1 / 2} I\left[\left(\frac{a}{b}\right)^{1 / 2}-\left(\frac{a}{b}\right)^{-5 / 2}+\left(\frac{a}{b}\right)^{-1 / 2}\left(\frac{H}{2 R_{0}}\right)^{-2}\right. \\
& \left.-\left(\frac{a}{b}\right)^{-3 / 2}\left(\frac{H}{2 R_{0}}\right)^{-2}\right]^{1 / 2} / \Sigma,
\end{aligned}
$$

where $E_{0}=\left[\sigma /\left(\varepsilon_{0} \varepsilon_{o} R_{0}\right)\right]^{1 / 2}$.

Equation (11) can be solved numerically for any initial separation of the droplets. Figure 3(a) shows the calculated relationship between $E$ and $a / b$ for both spheroidal and flattened droplets under $X_{0} / R_{0}=0.5$. The critical electric intensity $E_{c}$ to disintegrate the droplets is the maximum value of $E$. Obviously, $E_{c}$ for flattened droplets is larger than that for spheroidal droplets. To validate the above model, $E_{c} / E_{0}$ is calculated for different $X_{0} / R_{0}$ to compare with experimental data of Thiam et al. [18]. Note that $E$ in this paper refers to the electric intensity inside the continuous phase. The electric intensity in Ref. [18] should then be divided by the relative permittivity of the continuous phase $\varepsilon_{o}$. Figure $3(\mathrm{~b})$ shows the experimental boundaries between coalescence and noncoalescence for different $\mathrm{NaCl}$ concentrations. The boundaries are closed to each other owing to the large difference in conductivities of water and oil phases [17]. The calculated boundary shows a good agreement with the experimental results for

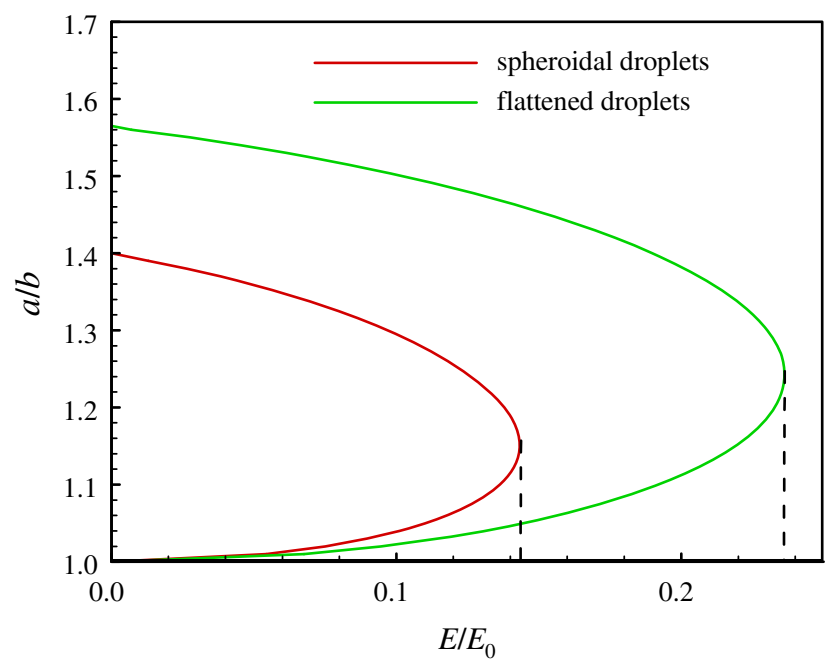

(a)
$X_{0} / R_{0}<0.7$, while disparities appear and increase for $X_{0} / R_{0}>0.7$. Interestingly, $X_{0} / R_{0}=0.7$ is near the boundary between coalescence and partial coalescence. Since partial coalescence happens when a sharp liquid cone forms, the disparities in Fig. 3(b) are likely attributed to the geometrical assumptions in the $x-z$ cross section, which are not valid for large deformations.

\section{Critical initial separation for partial coalescence}

The partial coalescence phenomenon observed in Ref. [18] is essentially controlled by the same mechanism of recoil phenomenon of the charged droplets [21]. Coalescence or recoil behavior of two droplets depends on the angle of the cones when the droplets just contact [21]. Figure 4(a) illustrates two droplets connected to a neck region. As mentioned in Sec. I, the comparison between the negative curvature $\left(1 / r_{n}\right)$ across the neck and the positive curvature $\left(1 / r_{p}\right)$ around the neck determines coalescence or recoil of the droplets [21]. Consequently, the transition between coalescence and recoil depends predominantly on the angle of the formed liquid cones [21]. We establish a geometrical model to relate the critical cone angle to the critical separation of a flattened droplet pair. Figure 4(b) shows the $x-y$ cross section when the tips of liquid cones just contact. Since the electric charges neutralize around the contact point, the surface tension dominates the flow of the connected portion. The cross sections of the droplets are assumed to have the same area as the initial separated droplets and the edges of the cones are tangential to a circle with the critical radius $R_{c}$. The relationship between $R_{c}$ and cone angle $\theta$ can be derived to be

$$
R_{c} / R_{0}=\sqrt{\frac{\pi}{\pi(1-\theta / 360)+1 / \tan (\theta / 2)}}
$$

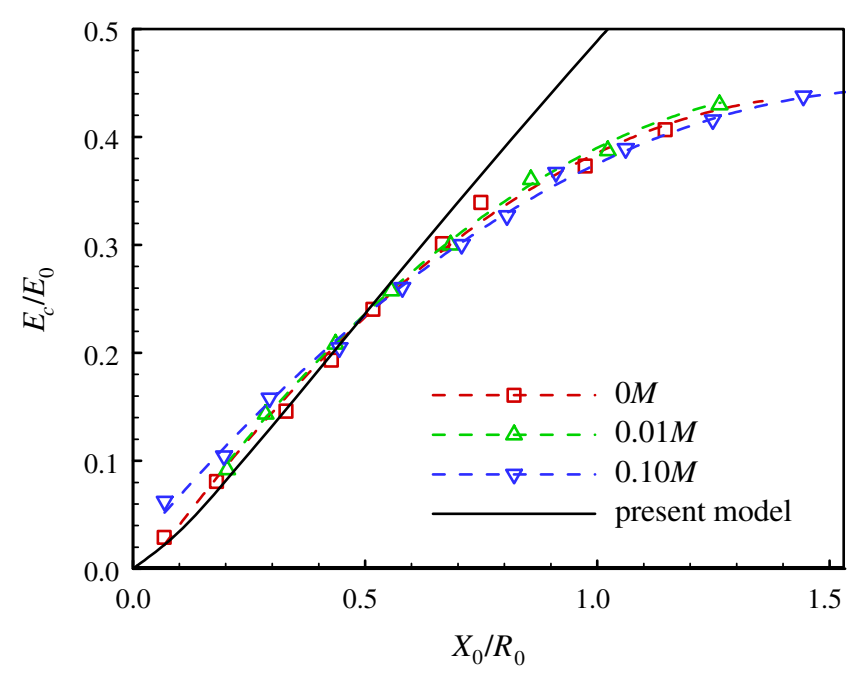

(b)

FIG. 3. (a) Relationship between electric intensities and deformations under $X_{0} / R_{0}=0.5$ for spheroidal and flattened droplet pairs. (b) Comparison of the present model and experimental data [18] for the boundary between coalescence and noncoalescence. 

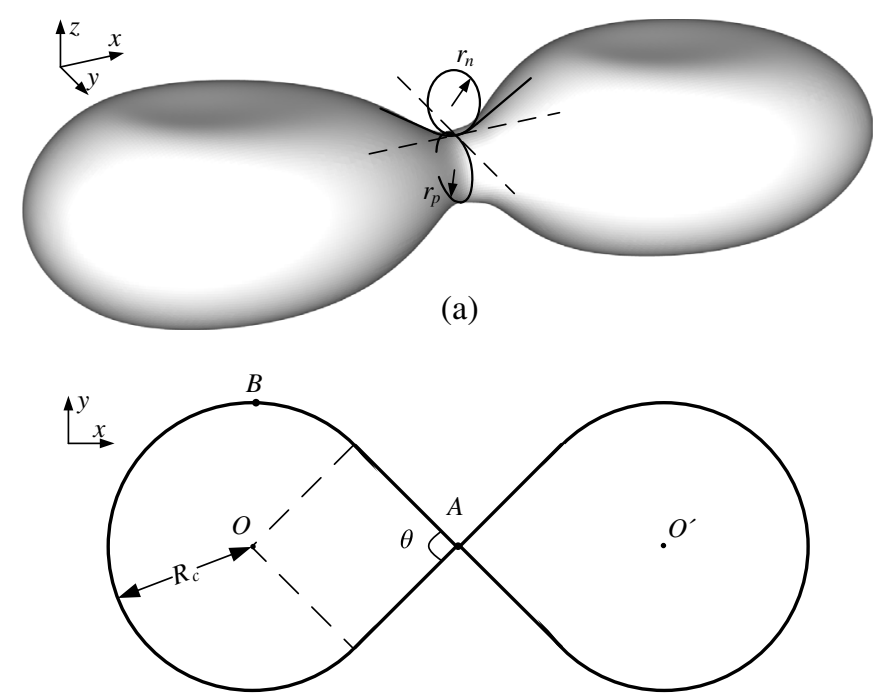

(b)

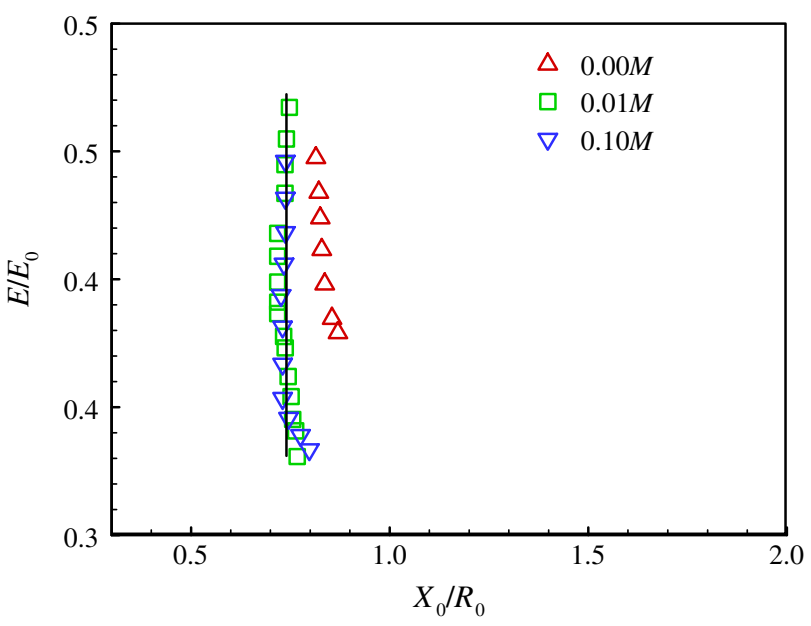

(c)

FIG. 4. (a) Two droplets connected with a neck regime with both positive and negative principal curvatures. (b) Schematic diagram of the critical condition in the $x-y$ cross section when the tips of liquid cones just contact. (c) Boundaries between coalescence and recoil for different $\mathrm{NaCl}$ concentrations from the experimental results in Ref. [18].

Since the droplet centroids do not change, the critical separation can be expressed as

$X_{c} / R_{0}=2 \sqrt{\frac{\pi}{\pi(1-\theta / 360)+1 / \tan (\theta / 2)}} / \sin (\theta / 2)-2$.

The theoretical prediction of the critical cone angle is not straightforward due to the complicated shape of the droplets. The boundary between coalescence and partial coalescence does not depend on the electric intensity as long as the droplets can make contact. The boundary should then be a vertical line on the regime diagram. Figure 4(c) shows the experimental boundaries between coalescence and partial coalescence extracted from the experimental results in Ref. [18]. As predicted theoretically, the boundaries are nearly straight vertical lines. The two boundaries for $\mathrm{NaCl}$ solutions are indistinguishable, while that of Milli-Q water is different from the others. This disparity is likely due to the large difference between the electrical conductivities of Milli-Q water and $\mathrm{NaCl}$ solutions. According to the experimental data in Fig. 4(c), the boundaries for $\mathrm{NaCl}$ solutions are at about $X_{0} / R_{0}=0.74$. The critical cone angle can be calculated from Eq. (6) to be about $90^{\circ}$. Note that Thiam et al. [18] showed a near-critical condition in their Fig. 2(b). The cone angle at $t=0.22 \mathrm{~ms}$ is very close to $90^{\circ}$. This means that Eq. (6) reveals the relationship between the critical separation and cone angle.

\section{NUMERICAL SIMULATION}

\section{A. Numerical methods}

We conduct direct numerical simulations to validate the models and show more details of the droplet deformation.
An open-source code GERRIS [28] with an electrohydrodynamic (EHD) extension [29] is adopted. The chargeconservative EHD scheme deals with two-phase problems using the volume-of-fluid (VOF) method and the leaky dielectric model. The VOF EHD method was proved to have the capability to describe EHD problems with large deformations, such as electrospray in the cone-jet mode [30] and the droplet-jet transition of electrified pendant droplets [31].

The formulation accommodates the conservation equations for an incompressible flow with surface tension and electric force [28],

$$
\begin{gathered}
\boldsymbol{\nabla} \cdot \boldsymbol{u}=0, \\
\rho\left(\partial_{t} \boldsymbol{u}+\boldsymbol{u} \cdot \boldsymbol{\nabla} \boldsymbol{u}\right)=-\boldsymbol{\nabla} p+\boldsymbol{\nabla} \cdot(2 \mu \boldsymbol{D})+\sigma \kappa \delta_{s} \boldsymbol{n}+\boldsymbol{F}_{e},
\end{gathered}
$$

where $\boldsymbol{u}$ is the velocity vector, $\rho$ is the fluid density, $\mu$ is the dynamic viscosity, and $\boldsymbol{D}$ is the deformation tensor defined as $D_{i j}=\left(\partial_{i} u_{j}+\partial_{j} u_{i}\right) / 2$. The Dirac delta function $\delta_{s}$ expresses the fact that the surface tension $\sigma$ is concentrated on the interface. The radius of curvature of the interface is denoted by $\kappa$, and $\boldsymbol{n}$ is the unit outward vector normal to the interface [28]. $\boldsymbol{F}_{e}$ is the volumetric electric force which can be derived from the electrostatic Maxwell stress tensor (by applying a divergence operator [29]) to be

$$
\boldsymbol{F}_{e}=\rho_{e} \boldsymbol{E}-\frac{1}{2} E^{2} \nabla \varepsilon
$$

where $\rho_{e}$ is the volumetric charge density, $\boldsymbol{E}$ is the electric field, and $\varepsilon$ is the electric permittivity. The two terms on the right-hand side represent the electric forces exerted on the free charges and the electric dipoles induced in dielectric mediums, respectively [29]. Note that the first term is 
zero in the present simulations since there is no impressed charge initially. The conservation equation for the volumetric charge density $q$ should be satisfied [29],

$$
\frac{\partial q}{\partial t}+\boldsymbol{u} \cdot \nabla_{s} q-q \boldsymbol{n} \cdot(\boldsymbol{n} \cdot \boldsymbol{\nabla}) \boldsymbol{u}+\|K \boldsymbol{E} \cdot \boldsymbol{n}\|=0,
$$

where $\nabla_{s}$ denotes the surface divergence and $K$ is the electric conductivity.

A VOF function $c(\boldsymbol{x}, t)$ is introduced to trace the interface. $c(\boldsymbol{x}, t)$ is defined as the volume fraction of a given fluid in each cell of the computational mesh. The density and viscosity can thus be written as

$$
\begin{aligned}
& \rho(c) \equiv c \rho_{w}+(1-c) \rho_{o}, \\
& \mu(c) \equiv c \mu_{w}+(1-c) \mu_{o} .
\end{aligned}
$$

According to mass continuity, the advection equation for the density takes the following form in terms of the volume fraction:

$$
\partial_{t} c+\nabla \cdot(c \boldsymbol{u})=0 .
$$

Similar to the fluid properties, the spatial values of the electrical properties can be interpolated from the volume fraction $c$,

$$
\begin{aligned}
& \varepsilon(c) \equiv c \varepsilon_{w}+(1-c) \varepsilon_{o} \\
& k(c) \equiv c k_{w}+(1-c) k_{o}
\end{aligned}
$$

A staggered temporal discretization of the volume fraction or density and pressure leads to a scheme which is second-order accurate in time [28]. A classical timesplitting projection method is utilized, which requires the solution of the Poisson equation. The time-stepping scheme combined with the discretization of the electric-field equation and the charge-evolution equation can be found in Ref. [29]. Spatial discretization is achieved using graded octree partitioning in three dimensions. All the variables are collocated at the center of each discretized cubic volume. Consistent with the finite-volume formulation, variables are interpreted as volume-averaged values for each cell. A piecewise-linear geometrical VOF scheme generalized for octree spatial discretization is used to solve the advection equation for the volume fraction [32]. The above numerical method allows for spatially and temporally varying resolution. In addition, local mesh refinement or coarsening in quadtree or octree discretization is cheap and can be performed at every time step, if necessary, with minimal impact on overall performance. Interpolation of quantities on refined or coarsened cells is also relatively simple on regular Cartesian mesh and is achieved conservatively both for momentum and volume fraction [28]. Several refinement criteria can be employed simultaneously, depending on the physical conditions encountered, to perform an adaptive mesh refinement [28] to the regions of interests.

\section{B. Model validation}

Numerical methods have been validated by our previous study on inertial migration of deformable droplets in a rectangular microchannel [33]. A validation of the EHD model is also performed (see the Supplemental Material [34]). The deformation of a leaky dielectric droplet in a leaky dielectric medium is considered following the extension [35] of Taylor's electrohydrodynamic theory [24] verified by experimental investigations [36]. The theory shows that the deformation parameter $D$ can be expressed as a function of the electric capillary number $\left(C_{E}=\varepsilon_{c} R_{0} E^{2} / \sigma\right)$, ratios of viscosities, conductivities, and electric permittivities between the droplet and ambient media. Eighteen conditions are simulated to consider the all the types of deformations and directions of flow motion. Good agreements are obtained with theoretical predictions for small $C_{E}$. Disparities at large $C_{E}$ are also observed because the theory is only valid for small deformation [37,38].

\section{Simulation setup}

A constant axial velocity is set to the left boundary to specify the flow. The right boundary has an outflow condition. No-slip conditions are set to the sidewalls. The aspect ratio of the cross section, $W / H$, is 3 . In the beginning, simulations proceed without droplets to obtain steady flow fields only with the continuous oil phase. The velocity profile relaxes to that of a rectangular Poiseuille flow in a noslip channel. Two cheese-wheel-like droplets are then placed along the centerline of the microchannel with an initial distance $X_{0}$. Simulations proceed without the electric field for $T$, which is long enough for the droplets to relax to steady state. With the presence of flow, thin oil films form between the droplet interfaces and the channel walls. The disjoining pressure inside the oil films avoids contact of the droplets and the walls. A sinusoidal electric potential $V$ is then applied across the computational domain from the right boundary to the left boundary. A no-penetration boundary condition of electric potential is enforced on the sidewalls.

\section{Grid considerations}

Two calculations are carried out to analyze the effect of grid resolution on the computational accuracy. The grids along the droplet interfaces are refined through a gradientbased refinement for the volume fraction to two different resolutions. Ratios of droplet radius $R_{0}$ and grid size $\Delta x$ are about 56 and 112, respectively. The background grid size is fixed to be $H / 16$ for both cases. Nearly identical evolutions of droplet separations and deformation are observed under the two resolutions (see the Supplemental Material [34]). The grid resolution with $R / \Delta x=56$ is accurate enough to be used in the remainder of this paper. 


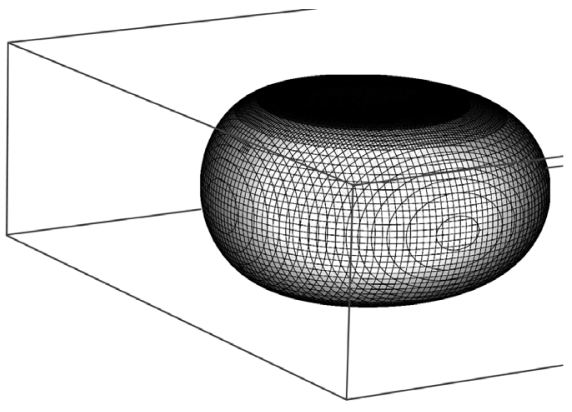

(a)

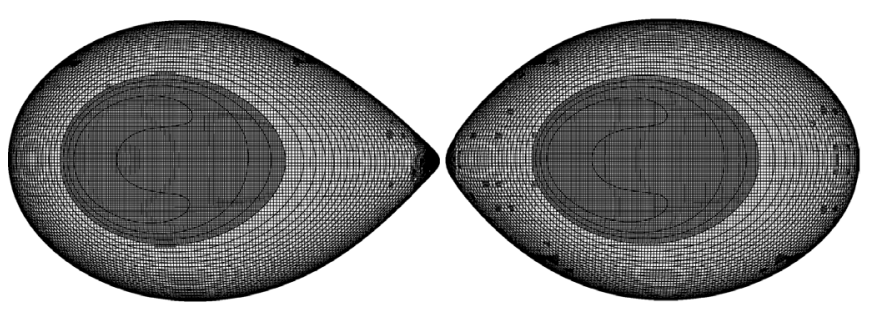

(c)

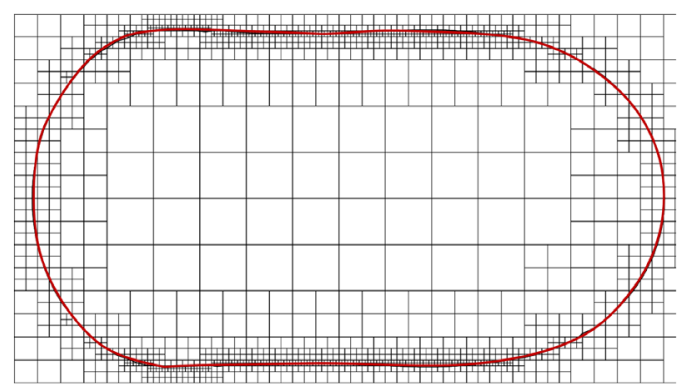

(b)

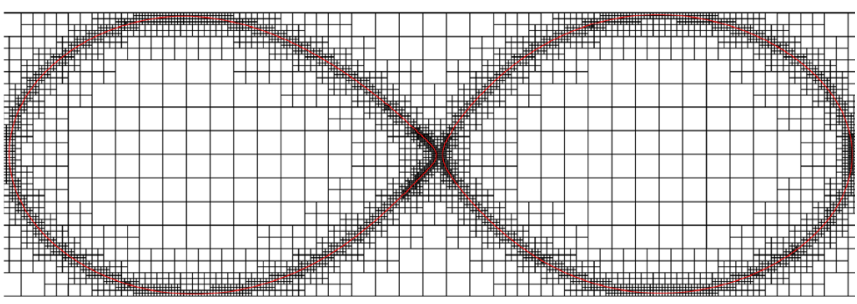

(d)

FIG. 5. (a) Droplet in a microchannel with a width-to-height ratio of 3 (the droplet surface is represented using VOF-reconstructed planar fragments in each interfacial cell). (b) Mesh in the $x-y$ cross section of (a). (c) Two droplets are in the process of contact at the tips. (d) Mesh in the cross section of (c).

Thickness-based and curvature-based refinement criteria are also used upon the abovementioned grid to further refine regions with thin and fine shapes. Thin liquid films exist between droplets and confinement walls. The thin films, however, are either not well resolved or avoided in existing numerical investigations in droplet microfluidics $[39,40]$. The distance-oriented thickness-based refinement criterion developed in our previous study [41] is used here to refine thin regions efficiently. The distance $d$ between the mass center of the interfacial grid and the wall is compared with the grid size $\Delta x$. If $d / \Delta x<4$, the interfacial grid is refined. In all the simulations, the disjoining pressure in thin liquid films is then directly simulated with at least two grids in the thickness direction. The interaction between droplet interfaces and the channel walls is well resolved. Figure 5(a) shows the VOF-reconstructed interface with the edges of interfacial cells of a flattened droplet. The interfacial cells near the wall are refined dynamically to resolve the thin film, which provides a cushion effect to separate the interface from the wall. Figure 5(b) displays the $x-z$ cross section of the computational mesh. The refinement level varies as needed to resolve the film thickness by at least two cells. The concave shapes of the interfaces near the confinement walls are well resolved with a smallest grid size of $R_{0} / 224$. On the other hand, sharp tips form along with the cone shapes when the droplets are close. A curvature-based refinement [28] is thus applied to improve the grid resolution of the tips. If $\kappa_{\max } \Delta x<1 / 5$, the interfacial grid is refined, where $\kappa_{\max }$ is the maximum curvature of the interface. Figure 5(c) shows two droplets that are about to contact through sharp tips. The meshes near the tips are refined dynamically according to the curvatures. Figure 5(d) highlights the mesh in the $x-y$ cross section. The curvature-based refinements only occur on the tips, to reduce the numerical cost considerably. The smallest grid size on the tips is $R_{0} / 896$.

\section{NUMERICAL RESULTS AND DISCUSSIONS}

\section{A. Regime diagram and predicted boundaries}

To obtain the whole regime diagram, total 32 simulation cases are performed for different $X_{0} / R_{0}$ and $E / E_{0}$. The simulations resolve the interaction of droplets, as well as thin films between the droplets and the walls during $1 \mathrm{~ms}$ (equivalent to 10T). Five types of phenomena are observed, namely noncoalescence, coalescence, partial coalescence, ejection after coalescence, and ejection with partial coalescence. Five movies showing different phenomena with photorealistic rendering [42] can be found in the Appendix, in Videos 1-5.

The droplets oscillate under the ac electric field, while the adjacent points are approaching gradually. If the electric intensity is smaller than the critical electric intensity for the initial separation, droplets cannot merge [see Fig. 6(a) and Video 1] in a desired duration. Otherwise, the deformation is large enough to make the droplets contact [see Fig. 6(b) and Video 2 in the Appendix]. Three-dimensional Taylor cones can be observed clearly in Fig. 6(b), when the interfaces of droplets are close enough $(t=1.50 T)$. The two tips are then connected to form a cylindrical liquid neck 


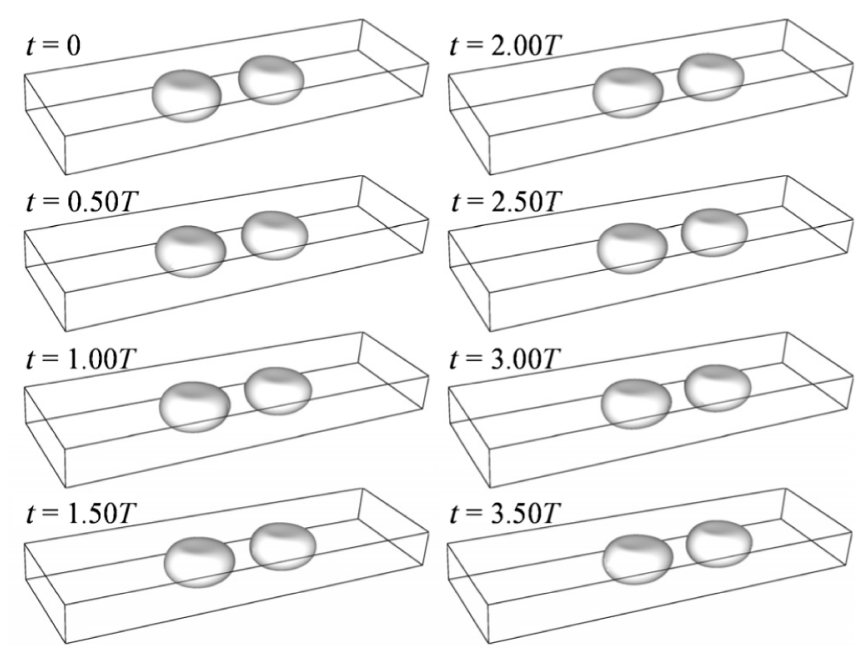

(a)

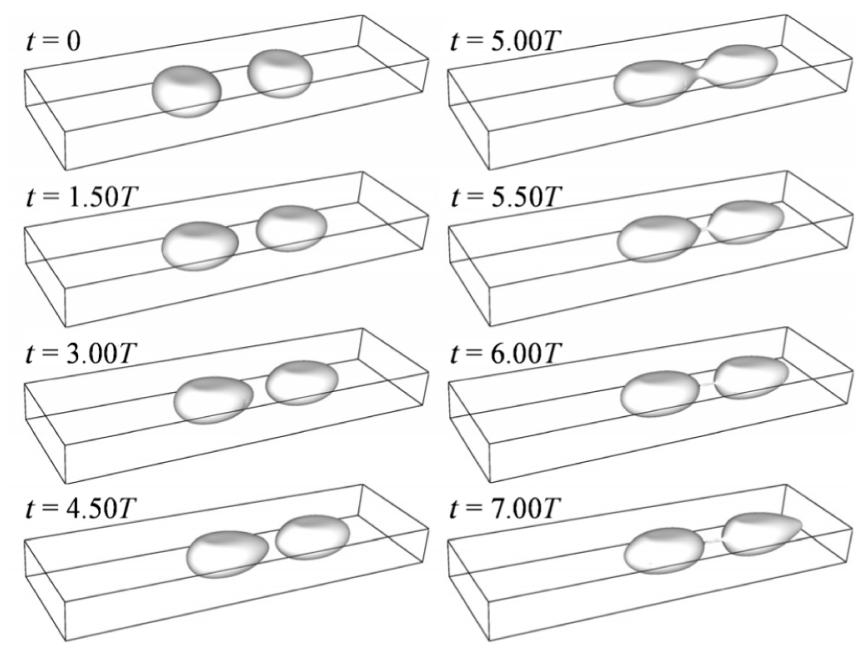

(c)

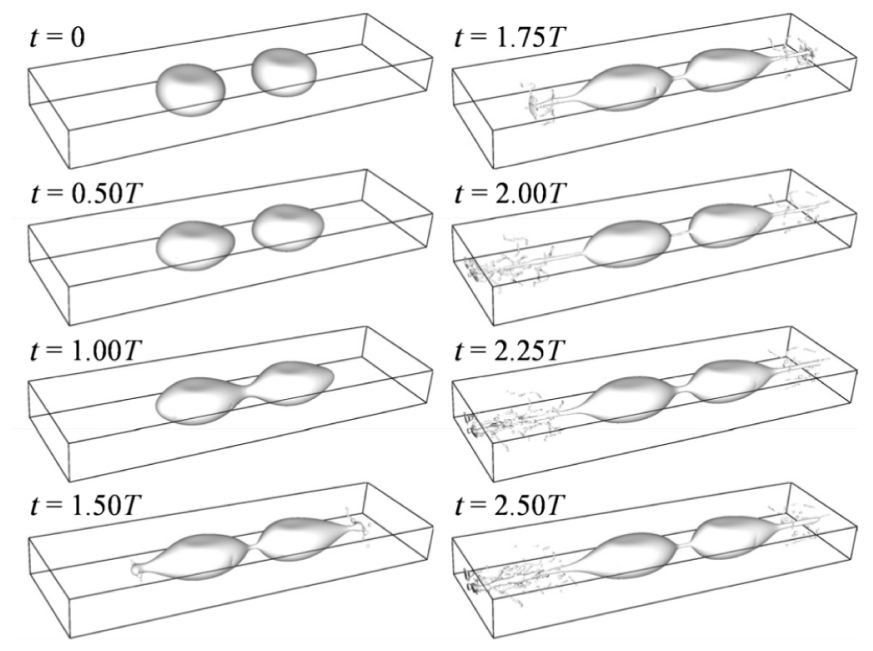

(e)

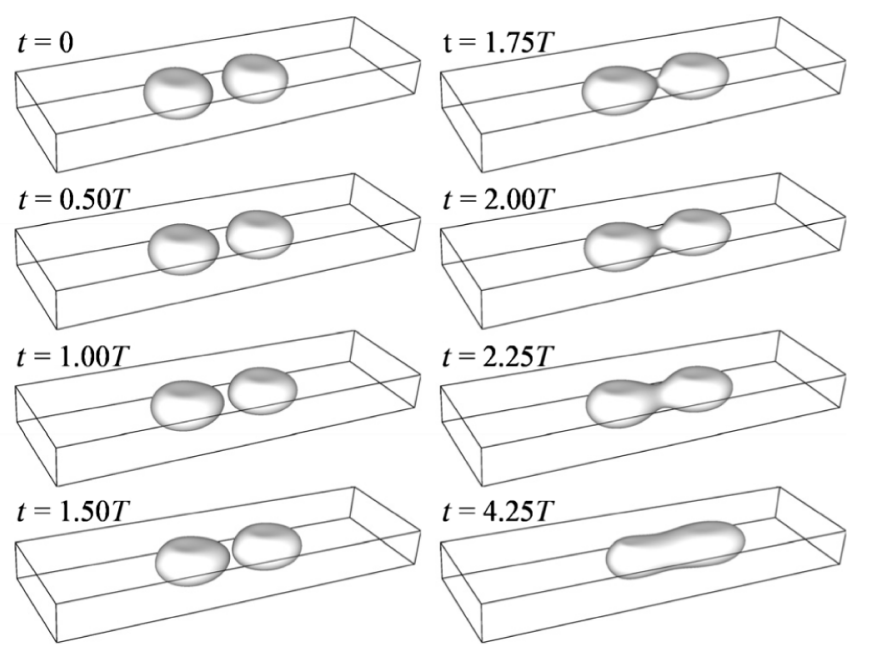

(b)

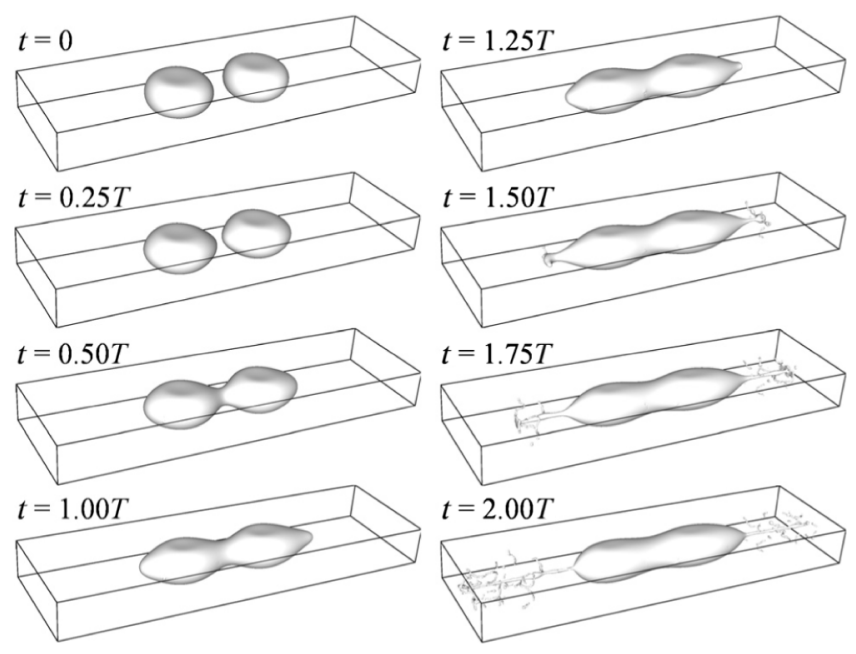

(d)

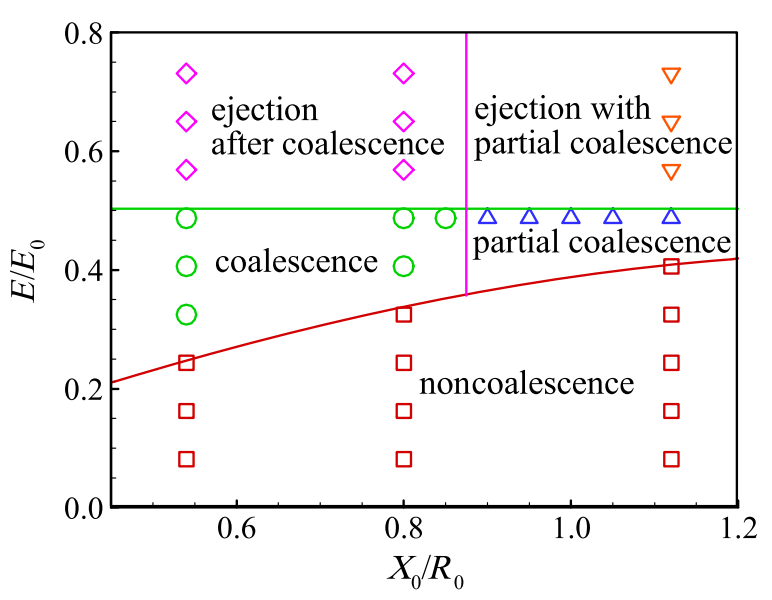

(f)

FIG. 6. (a) Noncoalescence $\left(E / E_{0}=0.32, X_{0} / R_{0}=0.80\right)$. (b) Coalescence $\left(E / E_{0}=0.41, X_{0} / R_{0}=0.54\right)$. (c) Partial coalescence $\left(E / E_{0}=0.49, X_{0} / R_{0}=1.12\right)$. (d) Ejection after coalescence $\left(E / E_{0}=0.65, X_{0} / R_{0}=0.54\right)$. (e) Ejection with partial coalescence $\left(E / E_{0}=0.65, X_{0} / R_{0}=1.12\right.$ ). (f) Region diagram of interactions of droplet pairs in terms of initial separation $X_{0} / R_{0}$ and ac electric intensity $E / E_{0}$. The five regions are noncoalescence $(\square)$, coalescence $(\bigcirc)$, partial coalescence $(\triangle)$, ejection after coalescence $(\diamond)$, and ejection with partial coalescence $(\nabla)$. For animations of the evolution of droplet deformation and interaction, see the Appendix, Videos 1-5. 
between the droplets. The neck expands radially to merge the droplets because the negative curvature across the neck is larger than the positive curvature around the neck. The droplets combine to become one prolate droplet at $t=4.25 T$. For a larger separation, the cone angle is also sharper, as shown in Fig. 6(c) at $t=5.00 T$. Since the negative curvature across the neck is smaller than the positive curvature around it, the neck shrinks and a fine liquid bridge forms between the droplets. The liquid bridge exists with the expanding-shrinking transition under the ac electric field (see Video 3 in the Appendix). This is the socalled partial coalescence [18]. Note that the liquid bridge can break to cause droplet recoil if the initial separation is near the critical value.

Similar to single droplets in a strong electric field, we find Taylor cones can form at the exterior ends (corresponding to point $D$ and $F$ in Fig. 2) of the droplet pair. Figure 6(d) shows cones appearing at the ends of the merged droplet at $t=1.00 T$. When the electric stress overcomes the capillary force at the tips of the cones, microjets emit from the exterior ends of the original droplets at $t=1.50 T$ (see Video 4 in the Appendix). The front of the jet is flattened by the surrounding flow with droplet shedding from the edge corners. This phenomenon can be called "ejection after coalescence." The overall dynamics are similar to electrospray in a cone-jet model, but the droplets loss volume and the steady state could not be reached. The emission of the microjets is also observed during partial coalescence as shown in Fig. 6(e) and Video 5 in the Appendix. This phenomenon is called "ejection with partial coalescence."

As shown in Fig. 6(f), the numerical simulations map all the five regimes in terms of the initial droplet separation and the electric intensity. The boundary of the noncoalescence regime is consistent with the curve obtained from the experimental results in Ref. [18]. The analysis in Sec. II C shows that the boundary between coalescence and partial coalescence is determined solely by a critical droplet separation. Five simulations with $X_{0} / R_{0}$ ranging from 0.80 to 1.12 are carried out for $E / E_{0}=0.49$ to find the critical separation. The boundary is approximately located at $X_{0} / R_{0}=0.88$, shown as a vertical line in Fig. 6(f). As discussed in Sec. II D, the boundary obtained from experimental data [18] is at $X_{0} / R_{0}=0.74$. This disparity may be attributed to the no-penetration boundary condition of electric potential on the side walls which is different from that in the experiments [18]. The issuing of a microjet at the rear ends of the droplet pair is comparable to that of a single droplet. The theoretical analysis of Taylor [11] shows that a droplet becomes disintegrated when $E(R / \sigma)^{1 / 2}>1.625$ (in electrostatic units), indicating that the boundary between stable and unstable conditions of the rear ends is a horizontal line in the regime diagram. $R$ should be replaced by the equivalent radius of a spherical droplet with the same volume as a flattened droplet. The calculated critical condition is then obtained in SI units to be $E_{c} / E_{0}=0.50$, which is in good agreement with present simulation results [as shown in Fig. 6(f)].

Overall, five regimes are separated by three boundariesan increasing curve between coalescence and noncoalescence, a vertical straight line between coalescence and partial coalescence, and a horizontal straight line between stable and unstable of the exterior ends. The regime diagram shows that coalescence happens only in a trapezoid region. The finding can be used to guide the selection and optimization of operational parameters of microfluidic devices for droplet fusion in the design stage. For example, the initial separation of the droplets should be smaller than the critical value of partial coalescence and the electric strength should be smaller than the critical value that causes tip streaming during the operation. The partial coalescence can be used in mass transfer between the droplets, especially when the droplets are of unequal size. The understanding of the interacting dynamics of the droplets can also help to realize other functions in microfluidic devices by changing the waveform and frequency.

\section{B. Droplet deformation and approaching}

Figures 7(a) and 7(b) show the calculated distributions of the electric field in the $x-y$ and $x-z$ cross sections, respectively. The contours are for axial electric intensity and isolines are for the electric potentials. As the electric charge relaxation time is much shorter than one half period of the voltage, the droplets can be regarded as equipotential [17]. Because the electric field is enhanced by the mutual interactions of the polarization charges, the maximum electric intensity in the $x$ direction locates at points $A$ and $C$. Figures 7(c) and 7(d) show the distribution of the electric forces acting on the interface. Since the dielectric permittivity and conductivity of water are much higher than those of oil, water can be considered to be perfectly conducting like metals. The electric stresses thus are almost perpendicular to the liquid surfaces. Figure 7(c) highlights that the forces at the two poles between the droplets are largest, but there is no force near the equators. On the other hand, as shown in Fig. 7(d), the forces are only located at the circular interfaces, while no force is observed at the flattened interfaces.

Figures 8 (a) and 8(b) show the evolutions of $X / R_{0}$ and $a / b$ for $X_{0} / R_{0}=0.54$ under different $E / E_{0}$, respectively. Since the electric field is strong and $X_{0} / R_{0}$ is small, the droplets interact quickly before their centroids move closer to each other. The changes of the distance of the droplet centroids are almost constant (varying only about $1 \%$ during $1 \mathrm{~ms}$; this was also observed in Ref. [18]). The relative movement of the mass center of droplets is thus negligible. As shown in Fig. 8(a), a full-period sinusoidal evolution is observed in each $T / 2$. For the noncoalescence outcome at $E / E_{0}=0.24, X / R_{0}$ decreases initially and then oscillates around $X / R_{0}=0.50$. For coalescence outcomes 


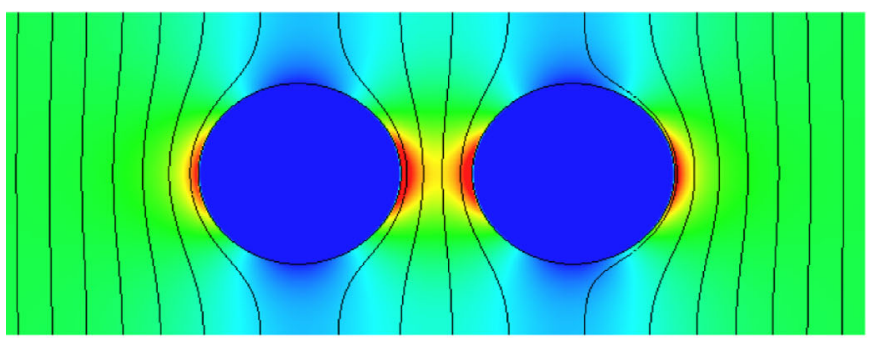

(a)

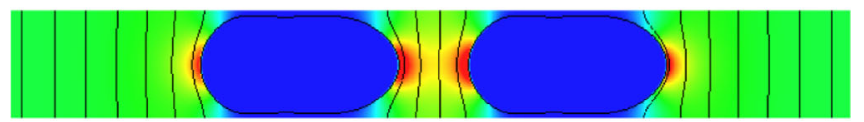

(b)

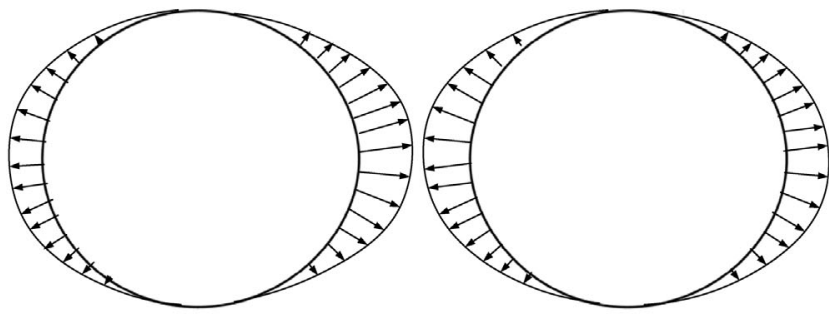

(c)

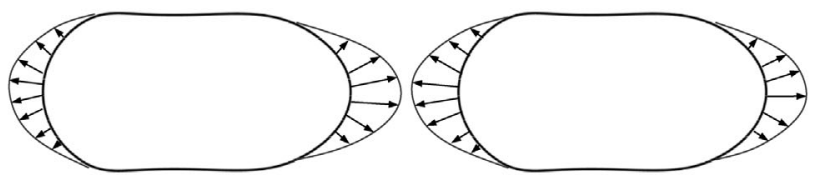

(d)

FIG. 7. Distribution of electric field near the droplet pair in the $x-y$ (a) and $x-z$ (b) cross section under $E / E_{0}=0.49$ and $X_{0} / R_{0}=1.12$. Electric force vectors on the interfaces in the $x-y$ (c) and $x-z$ (d) cross section.

at higher $E / E_{0}, X / R_{0}$ decreases linearly when the adjacent interfaces are far apart. When $X / R_{0}$ is less than $0.3, X / R_{0}$ decreases exponentially and reaches zero quickly. The evolutions of $a / b$ shown in Fig. 8(b) with solid lines are opposite to those of $X / R_{0}$.

To test the elliptical assumption used in Sec. II B, $a / b$ is expressed as a function of $X / R_{0}$ according to Eq. (6) to be

$$
a / b=\left(\frac{X_{0} / R_{0}-X / R_{0}+2}{2}\right)^{2}
$$

The dashed lines is Fig. 8(b) are the calculated $a / b$ according to Eq. (23), using the data of $X / R_{0}$ in Fig. 8(a).

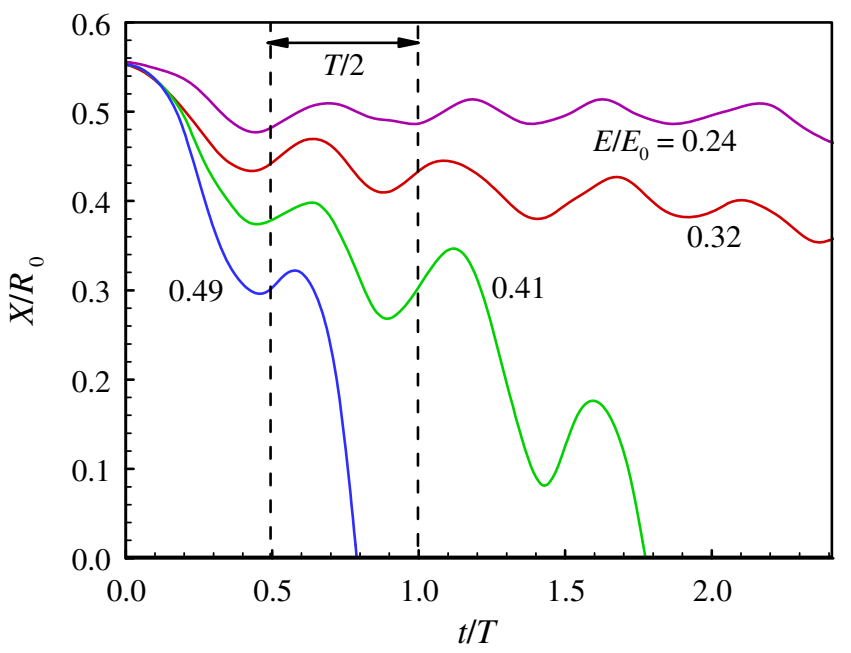

(a)
The reasonable agreements between the measured and calculated $a / b$ indicate that the elliptical assumption in the $x-y$ cross section is acceptable for analytical modeling.

Figure 9(a) shows droplet deformations just before and after the contact. The local refinement based on the curvature can resolve the liquid cones as sharp as possible. The cones penetrate the fluid between the droplets to make them contact. Since the droplets transfer with the surrounding media from left to right, the droplet shapes are influenced by the flow. The cone angle of the right droplet is larger than that of the left one. The cone angles are also different in the top and side views owing to the flattened droplet shape. The cone angle of the left droplet on the top

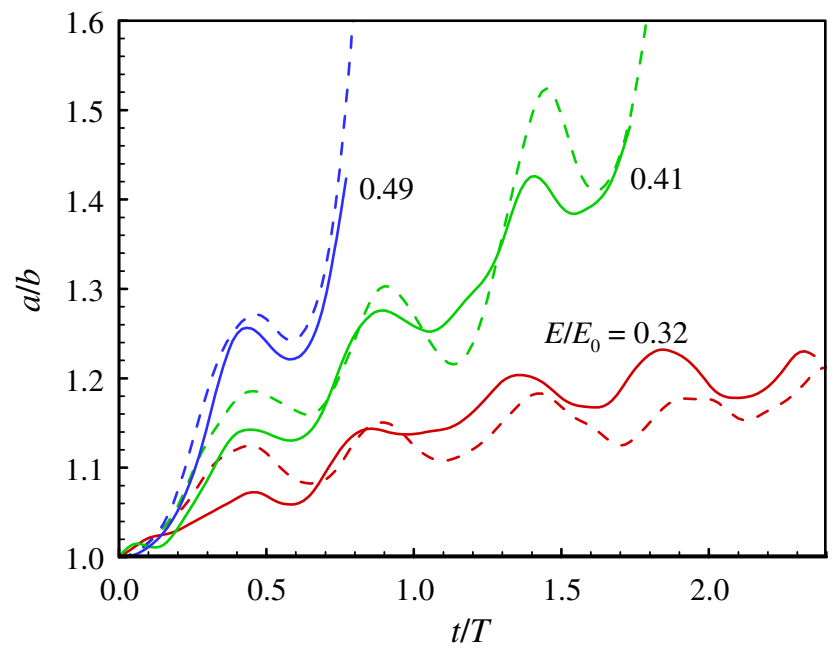

(b)

FIG. 8. Evolutions of droplet separation (a) and deformation (b) of a droplet pair under different electric intensities for $X_{0} / R_{0}=0.54$. 

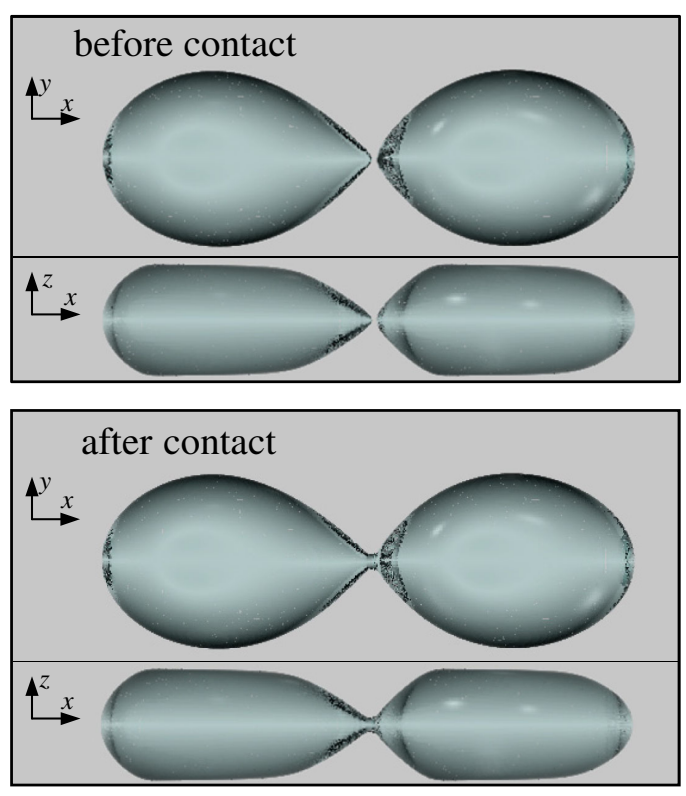

(a)

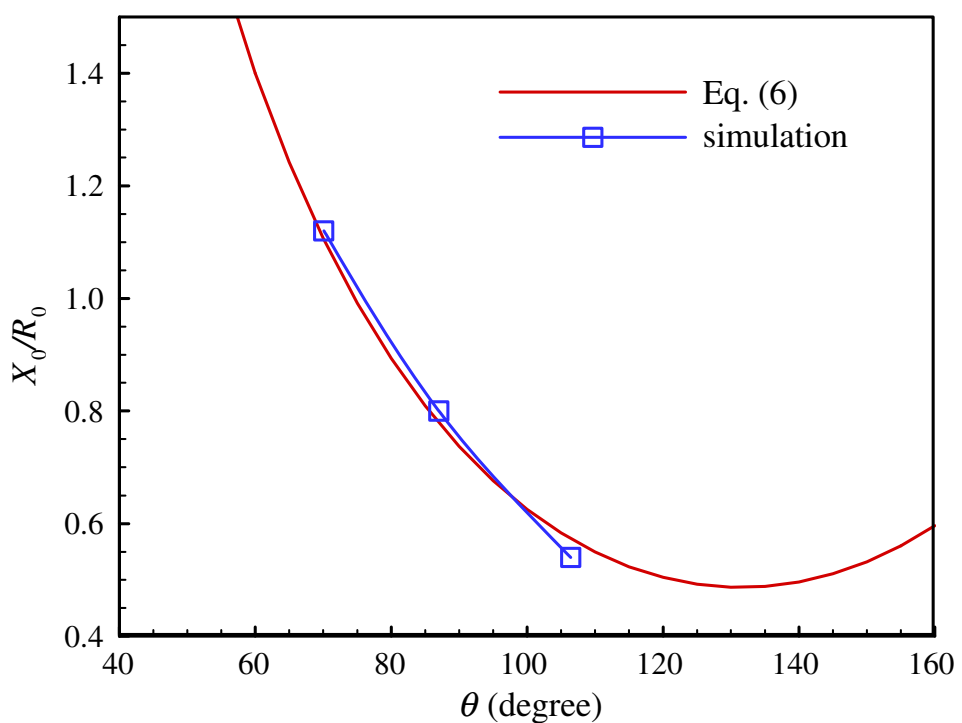

(b)

FIG. 9. (a) Photorealistic rendering of droplet pairs just before and after contact under $E / E_{0}=0.49$ and $X_{0} / R_{0}=0.80$. (b) Comparison of cone angles obtained from Eq. (6) and numerical simulations.

view is selected here to represent the cone shape. Independent of the electric intensity, the cone angles near the contact of the droplets are found to be solely determined by the initial separation. Figure 9(b) compares measured cone angles with the predictions from the geometrical model in Sec. II D, showing good agreements.

\section{Dynamics of the liquid bridge}

Visualizing the evolution of the liquid bridge between the droplets is difficult in experiments due to the relatively fast dynamics and small sizes. Current numerical simulations can capture the detailed dynamics and the underlying physics of the liquid bridge. Figure 10 shows the temporal evolution of the liquid bridge with photorealistic ray-tracing rendering. Prior to contact, droplets deform into cones at the near ends and oscillate under the ac electric field. The droplets merge to form a liquid bridge between them at $t=5.00 T$. The effect of surface tension turns to decrease the diameter of the liquid bridge and introduces higher capillary pressure to push the mass back to each of the droplets. Meanwhile, the electric force on the interface of the liquid bridge can be neglected since the liquid bridge is parallel to the ambient electric field. The diameter of the liquid bridge keeps decreasing until $t=6.00 T$. Surface tension dominates the dynamics of the liquid bridge from $t=5.00 T$ to $6.00 T$. The electric field then forces the droplets to transfer mass to the liquid bridge (with a maximum diameter at $t=6.25 T$ ). As the electric field reduces, the liquid bridge becomes fine again under the effect of surface tension. The diameter of the liquid bridge at $t=6.50 T$ is similar to that at $t=6.00 T$. The periodic evolution of the liquid bridge continues under the competition between the surface tension and electric stress.

Figure 11(a) shows the evolutions of minimum diameters of a liquid bridge under different electric intensities. The solid point at the beginning of each curve is at the moment when a liquid bridge forms. The diameter of the liquid bridge increases a little after formation due to the sudden change of the topology. The diameter then decreases to the minimum value under the capillary force. The diameter increases with increasing electric field. A local maximum of the diameter is reached near the peak of the electric field. The capillary force will again dominate the subsequent decreasing of the diameter. Larger oscillations of the diameter are observed for stronger electric fields. The average diameter of the liquid bridge also increases with the electric intensity. Figure 11(b) shows the evolution of the cone angle of the left droplet from the top view. The angle reduces a little after the merging of the droplets and then increases following the decreasing of the diameter of the liquid bridge. The local maximum value is achieved approximately when the electric intensity is zero. The angle then decreases when the electric intensity increases, and increases when the electric intensity decreases. Interestingly, the averaged half-angles of the cones are close to the $49.3^{\circ}$ of dc Taylor cones.

A more detailed shape evolution of the liquid bridge with high time resolution is shown in Fig. 12. After contact of the droplets, a liquid bridge with cylindrical shape forms under the effect of surface tension (at $t=6.7500 T$ ). The diameter of the liquid bridge decreases under the inward 


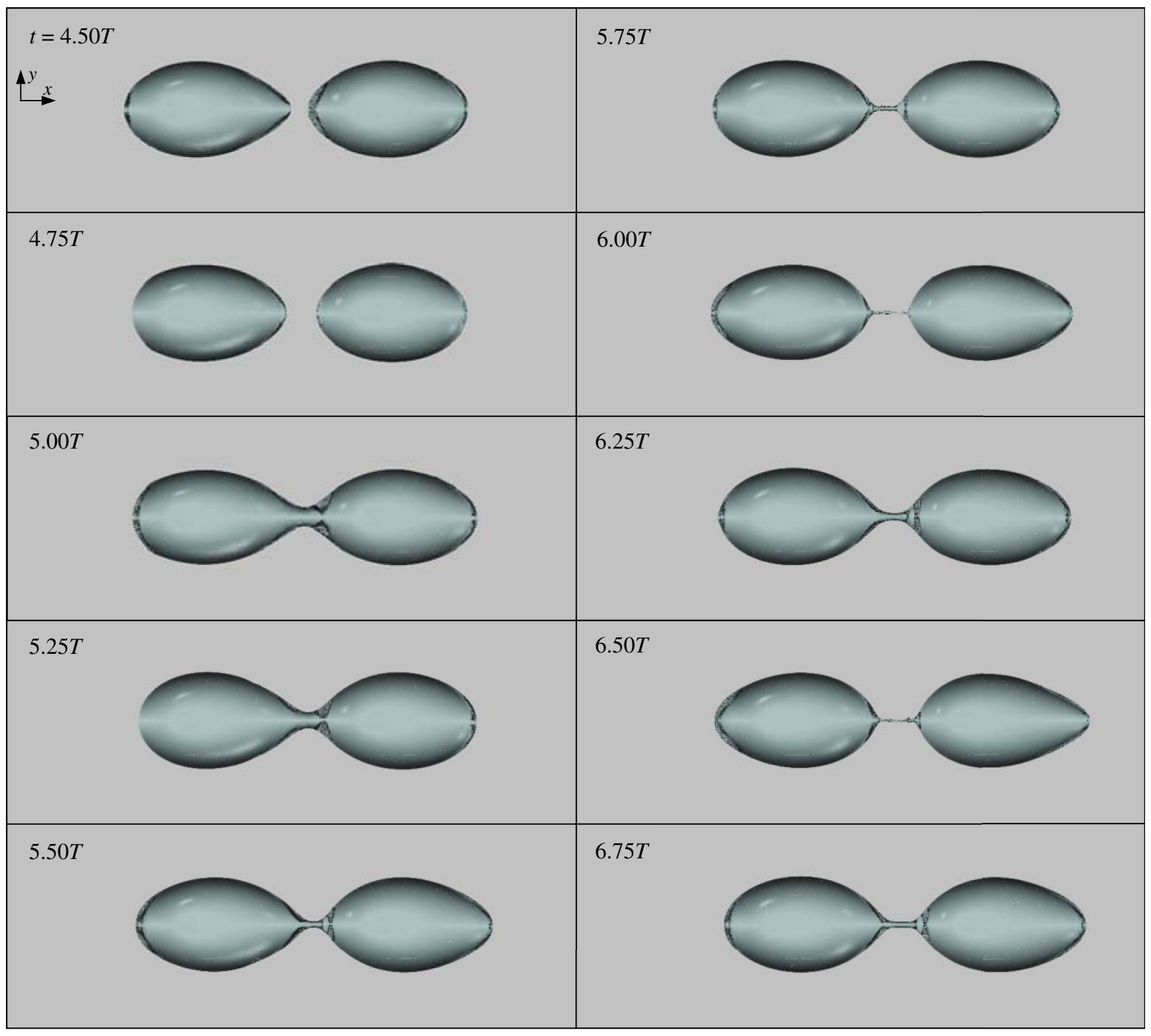

FIG. 10. Photorealistic rendering of partial coalescence under $E / E_{0}=0.49$ and $X_{0} / R_{0}=1.12$.

capillary force. The capillary pressure inside the liquid bridge is much larger than that in the droplets. The pressure gradient near the connect points between the droplets and the liquid bridge thus pushes the mass in the liquid bridge toward the droplets. This causes a fast decrease of the diameter of the liquid bridge near the connection points (as shown from $t=6.8125 T$ to $6.9375 T$ ), which is similar to the well-known "end-pinching" mechanism [43]. The slender threads connect the droplets and the spinningcone-like satellite droplet. If the electric field is weak, the satellite droplet is likely to transfer into a spherical shape to form droplet chains, as observed in electrostatic coalescers [17]. Note that the formation of a satellite droplet was actually captured experimentally in Ref. [18]. The tiny droplet in the center of the thread can be seen in the fifth snapshot in their Fig. 4. A clear ejection of droplets is observed at the points connecting with the fine liquid thread (at $t=7.000 T$ ). The jets emitted from the droplets connect with the satellite droplet at $t=7.1250 T$ to reform the liquid bridge. Meanwhile, the diameter in the center decreases because the electric field stretches the interface of the satellite droplet in the longitudinal direction. This stabilization effect of the electric field helps the liquid bridge against end pinching. Subsequently, a long liquid bridge with cylindrical shape reforms at $t=7.1875 T$ to complete the periodic process. Different from the statements in existing experimental studies $[17,18]$, our detailed simulations show that the existence of the liquid bridge is caused by the oscillating electric field, which competes with the capillary force to maintain the liquid bridge dynamically. 


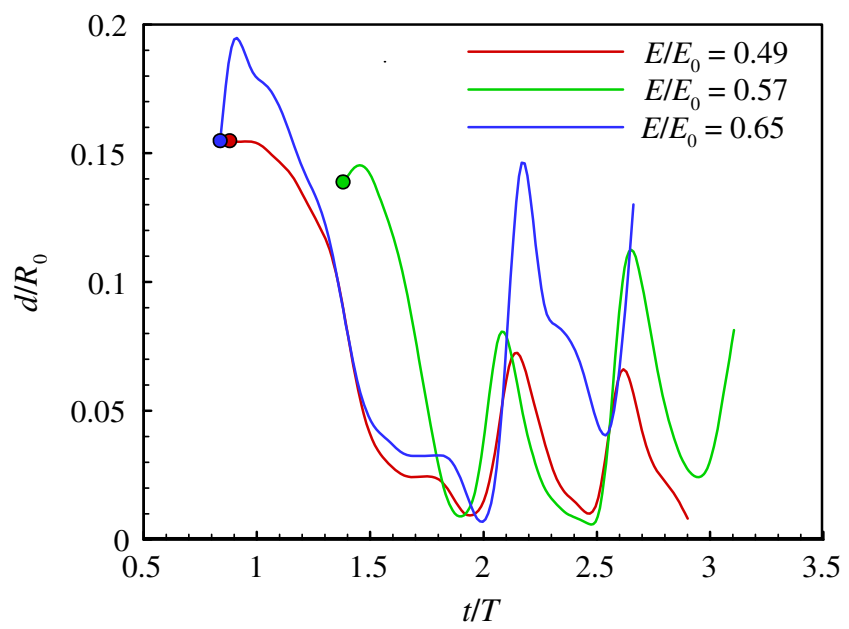

(a)

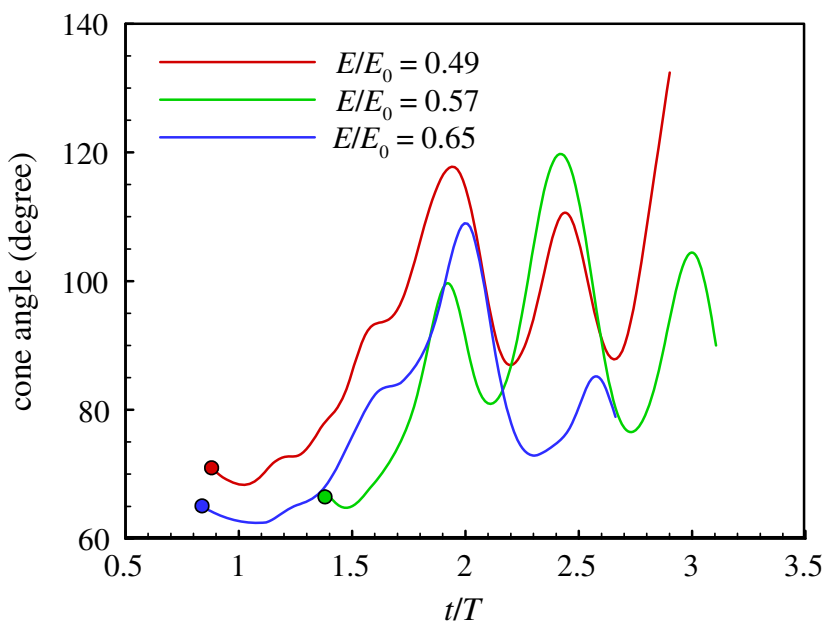

(b)

FIG. 11. Evolutions of minimum diameters (a) and cone angle (b) of liquid bridges under different electric intensities for $X_{0} /$ $R_{0}=1.12$. (Note that the horizontal coordinate for $E / E_{0}=0.49$ is subtracted by 4 to make it convenient to compare with other conditions.)

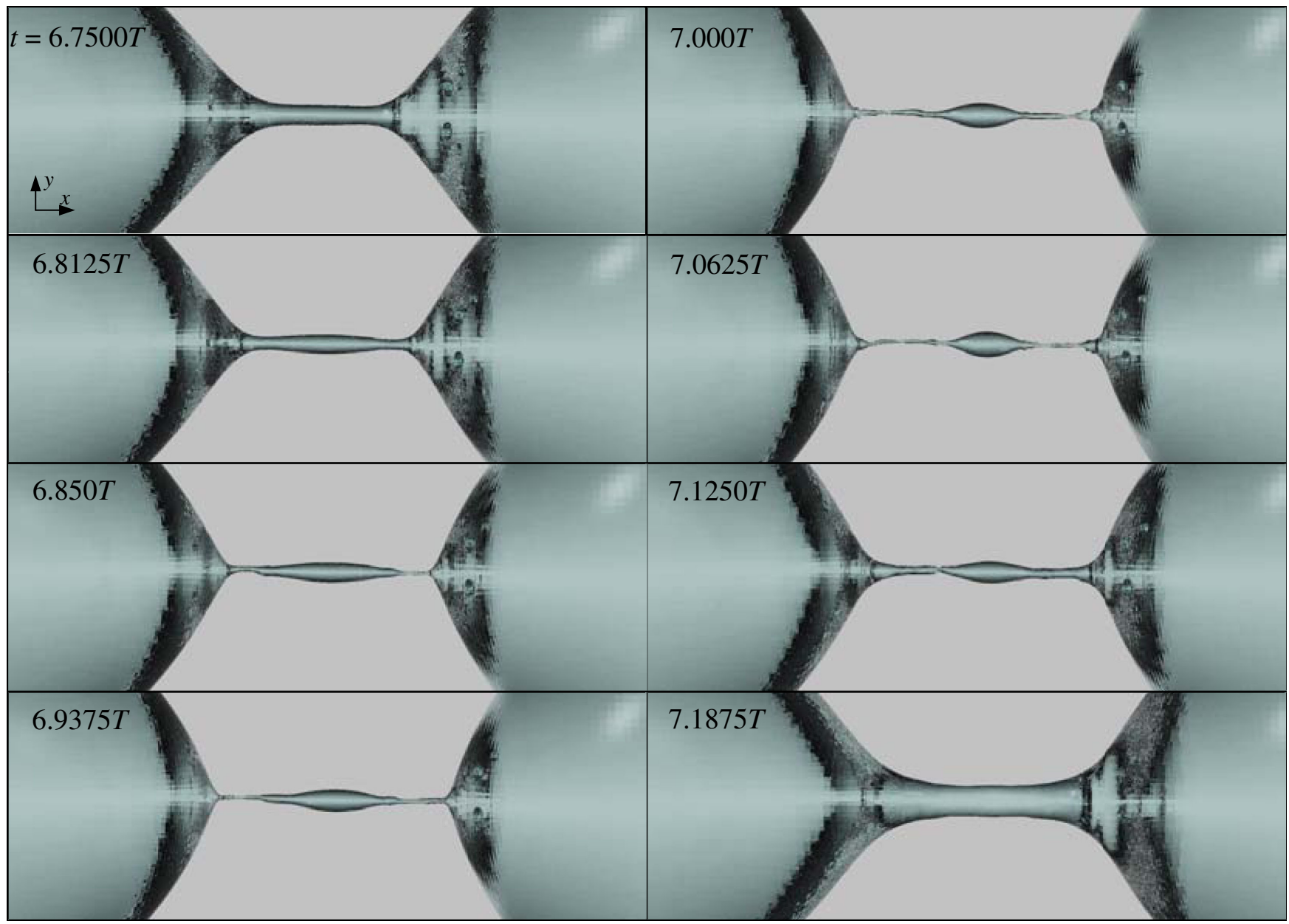

FIG. 12. Photorealistic rendering of the shape evolution of the liquid bridge under $E / E_{0}=0.49$ and $X_{0} / R_{0}=1.12$. 
In summary, the liquid bridge can be regarded to form when two Taylor cones emit against each other. The sustaining of the liquid bridge under a dc electric field is due to the competition between the surface tension and electric stress. Determined by the relationship of two principle curvatures of the liquid neck region [21], surface tension causes shrinking of the liquid bridge when the initial droplet separation exceeds a critical value. The electric field causes expanding of the liquid bridge by reforming and stabilizing the liquid bridge. Note that while a long liquid bridge under a dc electric field can be steady in shape and uniform in diameter [44], a liquid bridge under an ac electric field is unsteady in shape. From our numerical observation, the liquid bridge under an ac electric field changes from a thread with uniform diameter to a "beadon-a-string" structure, repeatedly.

\section{Dynamics of tip streaming}

The top panel in Fig. 13(a) shows a moment when tip streaming is just about to start at the ends of the coalesced droplets. Different from the sharp cones at the near ends of the droplets, the tips at the exterior ends are rounded. A fully developed jet is observed in the bottom panel of Fig. 13(a). Figure 13(b) shows the evolution of the angle of the right cone. The cone angle in the top view is larger than that in the side view due to the confined shape of the droplets. After the formation of the liquid jet, a full-period sinusoidal evolution is observed in each $T / 2$. At the moment marked with a red solid point, the tip streaming happens near the instant of the maximum electric intensity, corresponding to the moment shown in the top of
Fig. 13(a). The cone angle keeps reducing after emitting of the microjet. Shortly, the electric-induced deformation of the cone shape is balanced by the surface tension. The cone angle then increases to its maximum and a fully developed jet forms at the black point in Fig. 13(b), corresponding to the moment shown in the bottom of Fig. 13(a). The maximum and minimum cone angles happen at approximately $1 / 4$ and $3 / 4$ moment of a period. The average cone angle is about $53^{\circ}$ from the front view and about $46^{\circ}$ from the side view.

\section{CONCLUSION}

Deformation and interaction of droplet pairs in a microchannel under ac electric fields are investigated by means of a combined theoretical and numerical analysis. Theoretical models are established to predict the critical electric intensity for coalescence and the critical initial droplet separation for partial coalescence. Three-dimensional direct numerical simulations are preformed systematically to study the electrohydrodynamics in five different regimes, including, noncoalescence, coalescence, partial coalescence, ejection after coalescence, and ejection with partial coalescence. The three boundaries among the five regimes are defined, including an increasing curve between coalescence and noncoalescence, a vertical straight line between coalescence and partial coalescence, and a horizontal straight line between stable and unstable of the exterior ends. The detailed dynamics of the liquid bridge between the droplets, when the droplet separation exceeds the critical value, are resolved numerically. We show that sustaining of the liquid bridge under an ac electric field is
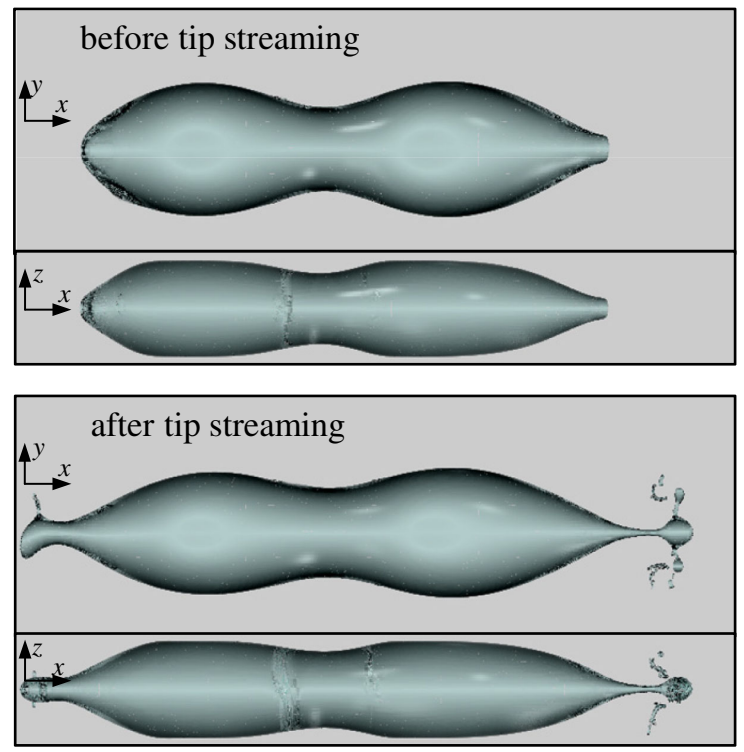

(a)

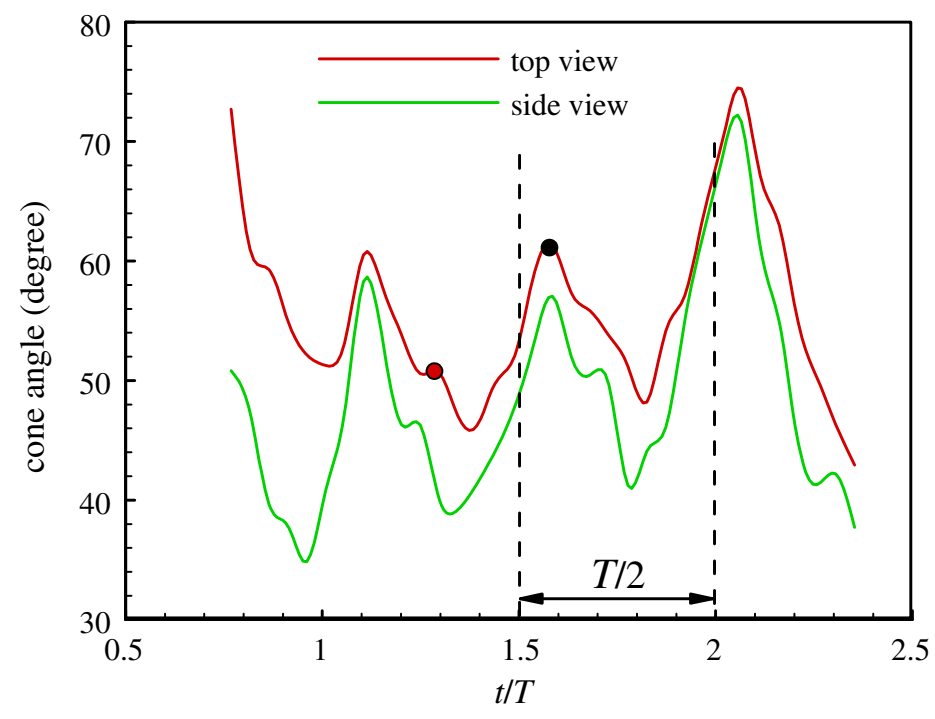

(b)

FIG. 13. (a) Photorealistic rendering of droplet pairs just before and after tip streaming under $E / E_{0}=0.41$ and $X_{0} / R_{0}=0.80$. (b) Evolutions of cone angles at the right end of the combined droplet. 
by the competition between the surface tension and electric stress in a dynamic manner. The liquid bridge changes from a thread with uniform diameter to a bead-on-a-string structure, repeatedly. The end-pinching phenomenon is also found during the capillary thinning of the liquid bridge. In addition, the dynamics of tip streaming is discussed to provide insight into the effect of the ac electric field. These understandings could be applied to the design and optimization of droplet fusion devices in microfluidics.

\section{ACKNOWLEDGMENTS}

This work was financially supported by MOST (Grant No. 2011CB707604) and NSFC (Grants No. 11402274 and No. 11272321). The authors would like to thank Dr. J. M. López-Herrera for helpful discussions.

\section{APPENDIX}

The dynamics of the deformation and interaction of the droplet pair in an ac electric field are demonstrated by

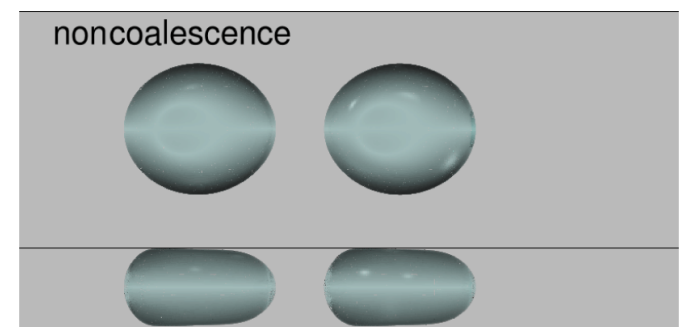

VIDEO 1. Noncoalescence $\left(E / E_{0}=0.32, X_{0} / R_{0}=0.80\right)$.

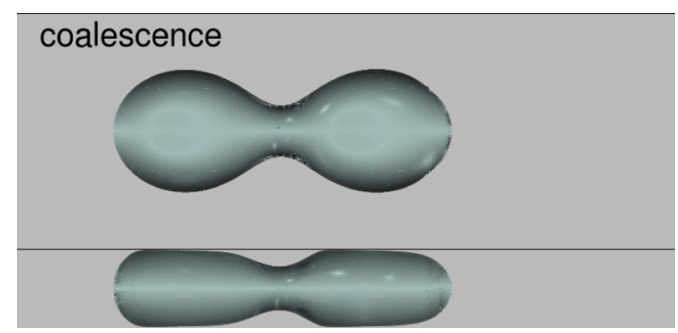

VIDEO 2. Coalescence $\left(E / E_{0}=0.41, X_{0} / R_{0}=0.54\right)$.

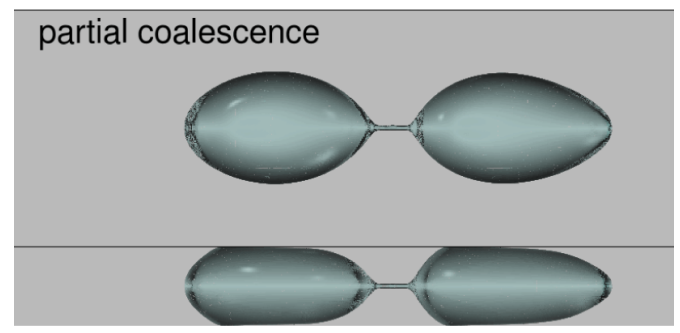

VIDEO 3. Partial coalescence $\left(E / E_{0}=0.49, X_{0} / R_{0}=1.12\right)$.

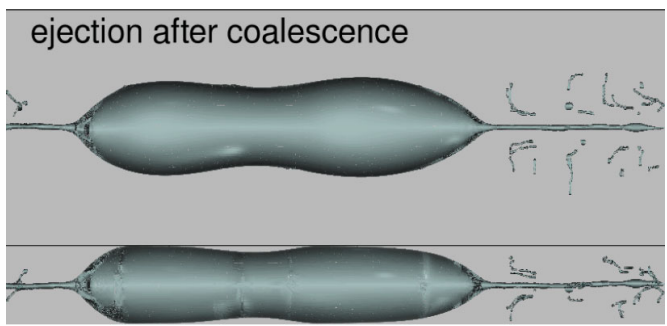

VIDEO 4. Ejection after coalescence $\left(E / E_{0}=0.65\right.$, $\left.X_{0} / R_{0}=0.54\right)$.

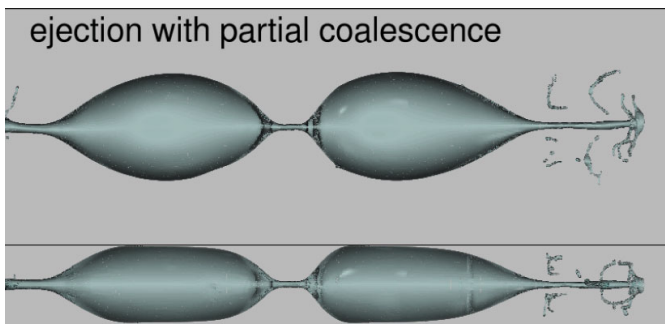

VIDEO 5. Ejection with partial coalescence $\left(E / E_{0}=0.65\right.$, $\left.X_{0} / R_{0}=1.12\right)$.

Videos 1-5 for the five phenomena, respectively, by photorealistic rendering [42]. Each video shows both top and side views of the droplet pair confined in the rectangular microchannel.

[1] A. B. Theberge, F. Courtois, Y. Schaerli, M. Fischlechner, C. Abell, F. Hollfelder, and W. T. Huck, Microdroplets in microfluidics: An evolving platform for discoveries in chemistry and biology, Angew. Chem., Int. Ed. 49, 5846 (2010).

[2] N. Bremond, A. R. Thiam, and J. Bibette, Decompressing Emulsion Droplets Favors Coalescence, Phys. Rev. Lett. 100, 024501 (2008).

[3] L. Mazutis and A. D. Griffiths, Selective droplet coalescence using microfluidic systems, Lab Chip 12, 1800 (2012).

[4] C. Priest, S. Herminghaus, and R. Seemann, Controlled electrocoalescence in microfluidics: Targeting a single lamella, Appl. Phys. Lett. 89, 134101 (2006).

[5] M. Chabert, K. D. Dorfman, and J. L. Viovy, Droplet fusion by alternating current (ac) field electrocoalescence in microchannels, Electrophoresis 26, 3706 (2005).

[6] L. Mazutis, J.-C. Baret, P. Treacy, Y. Skhiri, A. F. Araghi, M. Ryckelynck, V. Taly, and A. D. Griffiths, Multi-step microfluidic droplet processing: Kinetic analysis of an in vitro translated enzyme, Lab Chip 9, 2902 (2009).

[7] J. S. Eow and M. Ghadiri, Electrostatic enhancement of coalescence of water droplets in oil: A review of the technology, Chem. Eng. J. 85, 357 (2002).

[8] J. S. Eow, M. Ghadiri, A. O. Sharif, and T. J. Williams, Electrostatic enhancement of coalescence of water droplets in oil: A review of the current understanding, Chem. Eng. J. 84, 173 (2001). 
[9] M. Zagnoni and J. M. Cooper, On-chip electrocoalescence of microdroplets as a function of voltage, frequency and droplet size, Lab Chip 9, 2652 (2009).

[10] K. Ahn, J. Agresti, H. Chong, M. Marquez, and D. A. Weitz, Electrocoalescence of drops synchronized by size-dependent flow in microfluidic channels, Appl. Phys. Lett. 88, 264105 (2006).

[11] G. Taylor, Disintegration of water drops in an electric field, Proc. R. Soc. A 280, 383 (1964).

[12] J. Fernández de La Mora, The fluid dynamics of Taylor cones, Annu. Rev. Fluid Mech. 39, 217 (2007).

[13] J. Nolan, The breaking of water-drops by electric fields, Proc. R. Irish Acad., Sect. A 37, 28 (1924), http://www.jstor .org/stable/20490667.

[14] W. A. Macky, Some investigations on the deformation and breaking of water drops in strong electric fields, Proc. R. Soc. A 133, 565 (1931).

[15] J. Latham and I. W. Roxburgh, Disintegration of pairs of water drops in an electric field, Proc. R. Soc. A 295, 84 (1966).

[16] M. H. Davis, Two charged spherical conductors in a uniform electric field: Forces and field strength, Q. J. Mech. Appl. Math. 17, 499 (1964).

[17] L. Lundgaard, G. Berg, S. Ingebrigsten, and P. Atten, in Emulsions and Emulsion Stability (CRC Press, Boca Raton, 2006), p. 549.

[18] A. R. Thiam, N. Bremond, and J. Bibette, Breaking of an Emulsion under an ac Electric Field, Phys. Rev. Lett. 102, 188304 (2009).

[19] W. D. Ristenpart, J. Bird, A. Belmonte, F. Dollar, and H. Stone, Non-coalescence of oppositely charged drops, Nature (London) 461, 377 (2009).

[20] R. Allan and S. Mason, Particle motions in sheared suspensions. XIV. Coalescence of liquid drops in electric and shear fields, J. Colloid Sci. 17, 383 (1962).

[21] J. C. Bird, W. D. Ristenpart, A. Belmonte, and H. A. Stone, Critical Angle for Electrically Driven Coalescence of Two Conical Droplets, Phys. Rev. Lett. 103, 164502 (2009).

[22] P. Brazier-Smith, S. Jennings, and J. Latham, An investigation of the behaviour of drops and drop-pairs subjected to strong electrical forces, Proc. R. Soc. A 325, 363 (1971).

[23] C. Sozou, Electrohydrodynamics of a pair of liquid drops, J. Fluid Mech. 67, 339 (1975).

[24] G. I. Taylor, Studies in electrohydrodynamics. I. The circulation produced in a drop by electrical field, Proc. R. Soc. A 291, 159 (1966).

[25] J. C. Baygents, N. J. Rivette, and H. A. Stone, Electrohydrodynamic deformation and interaction of drop pairs, J. Fluid Mech. 368, 359 (1998).

[26] M. Mohammadi, S. Shahhosseini, and M. Bayat, Numerical study of the collision and coalescence of water droplets in an electric field, Chem. Eng. Technol. 37, 27 (2014).

[27] K. W. Yu and J. T. K. Wan, Interparticle force in polydisperse electrorheological fluids, Comput. Phys. Commun. 129, 177 (2000).
[28] S. Popinet, An accurate adaptive solver for surfacetension-driven interfacial flows, J. Comput. Phys. 228, 5838 (2009).

[29] J. M. López-Herrera, S. Popinet, and M. A. Herrada, A charge-conservative approach for simulating electrohydrodynamic two-phase flows using volume-of-fluid, J. Comput. Phys. 230, 1939 (2011).

[30] M. A. Herrada, J. M. López-Herrera, A. M. Gañán-Calvo, E. J. Vega, J. M. Montanero, and S. Popinet, Numerical simulation of electrospray in the cone-jet mode, Phys. Rev. E 86, 026305 (2012).

[31] C. Ferrera, J. M. López-Herrera, M. A. Herrada, J. M. Montanero, and A.J. Acero, Dynamical behavior of electrified pendant drops, Phys. Fluids 25, 012104 (2013).

[32] J. U. Brackbill, D. B. Kothe, and C. Zemach, A continuum method for modeling surface-tension, J. Comput. Phys. 100, 335 (1992).

[33] X. Chen, C. Xue, L. Zhang, G. Hu, X. Jiang, and J. Sun, Inertial migration of deformable droplets in a microchannel, Phys. Fluids 26, 112003 (2014).

[34] See Supplemental Material at http://link.aps.org/ supplemental/10.1103/PhysRevApplied.4.024005 for model validation and the grid independence study.

[35] O. Ajayi, A note on Taylor's electrohydrodynamic theory, Proc. R. Soc. A 364, 499 (1978).

[36] J. Melcher and G. Taylor, Electrohydrodynamics: A review of the role of interfacial shear stresses, Annu. Rev. Fluid Mech. 1, 111 (1969).

[37] E. Lac and G. M. Homsy, Axisymmetric deformation and stability of a viscous drop in a steady electric field, J. Fluid Mech. 590, 239 (2007).

[38] K. E. Teigen and S. T. Munkejord, Influence of surfactant on drop deformation in an electric field, Phys. Fluids 22, 112104 (2010).

[39] L. Wu, M. Tsutahara, L. S. Kim, and M. Ha, Threedimensional lattice Boltzmann simulations of droplet formation in a cross-junction microchannel, Int. J. Multiphase Flow 34, 852 (2008).

[40] M. M. Dupin, I. Halliday, and C. M. Care, Simulation of a microfluidic flow-focusing device, Phys. Rev. E 73, 055701 (2006).

[41] X. Chen and V. Yang, Thickness-based adaptive mesh refinement methods for multi-phase flow simulations with thin regions, J. Comput. Phys. 269, 22 (2014).

[42] Persistence of Vision Raytracer (version 3.7), http://www .povray.org/download/.

[43] H. A. Stone, Dynamics of drop deformation and breakup in viscous fluids, Annu. Rev. Fluid Mech. 26, 65 (1994).

[44] L. B. Skinner, C. J. Benmore, B. Shyam, J. Weber, and J. B. Parise, Structure of the floating water bridge and water in an electric field, Proc. Natl. Acad. Sci. U.S.A. 109, 16463 (2012). 